\title{
Lung epithelium and myeloid cells cooperate to clear acute pneumococcal infection
}

\author{
M Dudek ${ }^{1}$, F Puttur ${ }^{1}$, C Arnold-Schrauf ${ }^{1}$, AA Kühl ${ }^{2}$, B Holzmann ${ }^{3}$, B Henriques-Normark ${ }^{4,5}$,

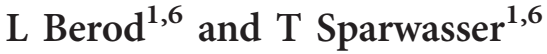

The Gram-positive bacterium Streptococcus pneumoniae causes life-threatening infections, especially among immunocompromised patients. The host's immune system senses $S$. pneumoniae via different families of pattern recognition receptors, in particular the Toll-like receptor (TLR) family that promotes immune cell activation. Yet, while single TLRs are dispensable for initiating inflammatory responses against $S$. pneumoniae, the central TLR adapter protein myeloid differentiation factor 88 (MyD88) is of vital importance, as MyD88-deficient mice succumb rapidly to infection. Since MyD88 is ubiquitously expressed in hematopoietic and non-hematopoietic cells, the extent to which MyD88 signaling is required in different cell types to control S. pneumoniae is unknown. Therefore, we used novel conditional knockin mice to investigate the necessity of MyD88 signaling in distinct lung-resident myeloid and epithelial cells for the initiation of a protective immune response against $S$. pneumoniae. Here, we show that MyD88 signaling in lysozyme M (LysM)- and CD11c-expressing myeloid cells, as well as in pulmonary epithelial cells, is critical to restore inflammatory cytokine and antimicrobial peptide production, leading to efficient neutrophil recruitment and enhanced bacterial clearance. Overall, we show a novel synergistic requirement of compartment-specific MyD88 signaling in S. pneumoniae immunity.

\section{INTRODUCTION}

Streptococcus pneumoniae is a Gram-positive bacterium that frequently causes invasive diseases such as acute pneumonia, sepsis, and meningitis in infants, the elderly, and immunocompromised individuals. ${ }^{1}$ An essential prerequisite for invasive pneumococcal infection of the lung involves the microaspiration of S. pneumoniae into the airway lumen. Upon entry into the lung, invading bacteria encounter the first line of defense, composed of the lung epithelium, alveolar macrophages (AMs), and dendritic cells (DCs). These cells recognize $S$. pneumoniae via pathogen recognition receptors like Toll-like receptors (TLRs). ${ }^{2}$ Bacterial cell wall and membrane components such as peptidoglycan, lipoproteins, and lipoteichoic acid serve as TLR2 ligands, ${ }^{3,4}$ whereas intracellularly located unmethylated CpG-containing DNA is recognized by TLR9. ${ }^{5,6}$ Moreover, S. pneumoniae-secreted toxins like the hemolytic pneumolysin, a key virulence factor, activate TLR4. $^{7,8}$

TLR activation in AMs induces several effector functions against $S$. pneumoniae infection to contain and eradicate bacteria, including phagocytosis, secretion of proinflammatory cytokines, and the induction of apoptosis. ${ }^{9}$ One of the first proinflammatory cytokines released upon $S$. pneumoniae infection is tumor necrosis factor $\alpha(\mathrm{TNF} \alpha)$, which acts in concert with the chemoattractants CXCL1 (KC, keratinocyte chemoattractant) and CXCL2 (MIP-2, macrophage inflammatory protein 2) to recruit polymorphonuclear neutrophils (PMNs) from the periphery to the lungs. ${ }^{10-13}$ PMNs efficiently eradicate pneumococci, especially during acute pneumonia when increased bacterial proliferation supersedes the phagocytic capacity of AMs. ${ }^{14}$ To this end, PMNs engulf bacteria into the phagolysosome followed by the

${ }^{1}$ Institute of Infection Immunology, TWINCORE, Centre for Experimental and Clinical Infection Research, a joint venture between the Medical School Hannover (MHH) and the Helmholtz Centre for Infection Research (HZI), Hannover, Germany. ${ }^{2}$ Department of Medicine I for Gastroenterology, Infectious Disease and Rheumatology, Campus Benjamin Franklin, Charité-Universitätsmedizin, Berlin, Germany. ${ }^{3}$ Department of Surgery, Technische Universität München, Munich, Germany. ${ }^{4}$ Department of Microbiology, Tumor and Cell Biology, Karolinska Institutet, Stockholm, Sweden and ${ }^{5}$ Department of Clinical Microbiology, Karolinska University Hospital, Stockholm, Sweden. Correspondence: T Sparwasser (Sparwasser.office@mh-hannover.de)

${ }^{6}$ These authors contributed equally to this work.

Received 1 July 2015; accepted 1 November 2015; published online 2 December 2015. doi:10.1038/mi.2015.128 
subsequent exposure to antimicrobial peptides. ${ }^{15}$ In contrast to AMs and PMNs, the phagocytic and bactericidal capability of DCs is reduced. ${ }^{16}$ During infection, resident DCs within the pulmonary interstitial spaces extend their protrusions through the lung epithelium into the alveolar lumen to sample antigens and pathogens. ${ }^{17}$ Upon antigen encounter, DC subsets such as $\mathrm{CD}_{103}{ }^{+}$DCs preferentially migrate to lung-draining lymph nodes (LNs) in a CCR7-dependent manner. DCs pulsed with intact pneumococci are potent activators of the adaptive immune system. ${ }^{18}$

Besides innate immune cells, the lung epithelium consisting of several specialized cell types also expresses TLRs and contributes to innate immune responses. ${ }^{19}$ Club cells (CCs, formerly known as Clara cells) are lung epithelial cells expressing TLR4, among other pathogen recognition receptors, that line the bronchiolar airways down to the alveoli, where S. pneumoniae preferentially causes invasive disease. ${ }^{20}$ Hence, CCs can be triggered to secrete inflammatory cytokines along with antimicrobial peptides, but their role in pneumococcal defense in vivo remains elusive. ${ }^{21,22}$ Surfactant protein D (SP-D), expressed by alveolar type II cells and CCs, is important for surfactant homeostasis and also serves as an antimicrobial peptide. $^{23-25}$ Furthermore, patients with SP-D deficiency or genetic polymorphisms are more prone to recurrent pneumonia compared to control patients. ${ }^{26,27}$

All these different cell types share common TLR expression in order to exert specific antibacterial functions. However, the activation of individual TLRs has a limited relevance in $S$. pneumoniae immunity as suggested by single TLR-deficient mice, which are able to clear the infection. ${ }^{8,28,29}$ Myeloid differentiation factor 88 (MyD88) is the central signal transduction protein for most TLRs, except for TLR3 and partially for TLR4, and is required for the activation and translocation of nuclear factor- $\mathrm{\kappa B}$ into the nucleus and the induction of proinflammatory gene expression. In addition to TLR, interleukin-1 receptor (IL-1R) signaling is also mediated by MyD88 but similar to single TLR deficiency negligible in pneumococcal immunity. ${ }^{10,30}$ Mice deficient in MyD88 $\left(\mathrm{MyD} 8^{-1-}\right)$ display impaired innate immune responses reflected by the absence or low levels of proinflammatory cytokines and phagocytic cells, and hence increased bacterial burden. In addition, MyD88 ${ }^{-1-}$ mice are highly susceptible to S. pneumoniae and die early after infection. ${ }^{31}$ Thus, during pneumococcal infection, innate signaling via MyD88 is essential. Yet, despite being the fact that complete deficiency of MyD88 strongly reduces antibacterial responses against S. pneumoniae, the precise contribution of cell type-specific MyD88 signaling provided by hematopoietic and lung epithelial cells in the control of pneumococci remains unclear. To date, the lack of transgenic mouse models for specifically targeting MyD88 in distinct cell subsets has constrained our understanding of MyD88 signaling in innate immune responses against $S$. pneumoniae and other pathogens. ${ }^{32}$

In this study, we made use of novel mouse models in which MyD88 expression is restricted to myeloid-derived or lung epithelial cells. We demonstrate that MyD88 signaling in AMs,
DCs, and PMNs is crucial for initiating proinflammatory cytokine release and subsequent bacterial eradication, whereas MyD88 signaling in CCs is needed for the enhanced production of antimicrobial peptide to restrict bacterial outgrowth. Our results show that the concerted action of hematopoietic and lung epithelial cells, via MyD88 signaling, is essential for protective immune responses against $S$. pneumoniae.

\section{RESULTS}

\section{Complete protection against pneumococcal infection requires MyD88 signaling in hematopoietic and non- hematopoietic cells}

To address the precise role of MyD88-dependent immune responses derived from the hematopoietic and non-hematopoietic compartment, we first generated wild type (WT) and ${\mathrm{MyD} 88^{-1-}}^{-1}$ bone marrow (BM) chimeric mice. The degree of chimerism after lethal irradiation and BM transfer was monitored (except for the MyD88 group) using WT mice expressing the congenic marker Ly5.1 either as BM donor $\left(\right.$ Ly5.1 $\rightarrow$ MyD88 $^{-l^{-}}$) or recipient $\left(\mathrm{WT} \rightarrow\right.$ Ly5.1, MyD88 ${ }^{-1}$ $\rightarrow$ Ly5.1). First, we evaluated disease survival to determine whether compartment-specific MyD88 signaling is sufficient to rescue mice from S. pneumoniae infection. For this purpose, we used the S. pneumoniae TIGR4 strain, commonly found in patients with invasive infection. ${ }^{33}$ Furthermore, the kinetics of bacterial dissemination was followed using the isogenic luciferase-expressing TIGR4X strain ${ }^{31}$ and the in vivo imaging system (IVIS) Spectrum computer tomograph (CT) imager (Supplementary Figure S1 online). BM-reconstituted mice were inoculated intranasally with $0.2 \times 10^{6}$ colony-forming units (CFUs) of S. pneumoniae (termed low dose, since higher dosages caused death in $>30 \%$ of WT mice; Supplementary Figure S2a), and survival was assessed for $336 \mathrm{~h}$ (14 days). Consistent with previous findings, ${ }^{31}$ our results show that $90 \%$ of the mice fully sufficient for MyD88 signaling (Ly5.1 $\rightarrow$ WT) survived the infection, whereas all MyD88-deficient mice $\left(\mathrm{MyD} 88^{-/-} \rightarrow \mathrm{MyD}^{-1-}\right.$ ) succumbed before $85 \mathrm{~h}$ (Figure 1a and Supplementary Figure S1). Interestingly, mice expressing MyD88 only in hematopoietic

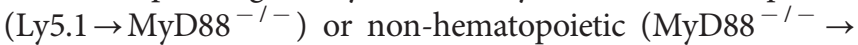
Ly5.1) cells showed a survival rate of $\sim 40 \%$, indicating that MyD88 signaling in either the hematopoietic or non-hematopoietic compartment critically contributes to the protective response against $S$. pneumoniae infection.

To further investigate the role of MyD88 signaling during $S$. pneumoniae infection, we analyzed inflammatory cytokine production as well as PMN infiltration of low-dose-infected mice $18 \mathrm{~h}$ post infection (p.i.) WT and $\mathrm{MyD} 88^{-1-}$ mice showed no significant differences in lung TNF $\alpha$, IL-6, or total PMN numbers, although at this time point bacterial burden was significantly increased in MyD88-deficient mice compared to WT mice (Supplementary Figure S2b). To examine if this phenotype was influenced by the dose of inoculum, BM chimeric mice were infected with $2 \times 10^{6}$ CFUs of $S$. pneumoniae (termed high dose), and the number of CFUs in lung homogenates was analyzed. Bacterial loads in 

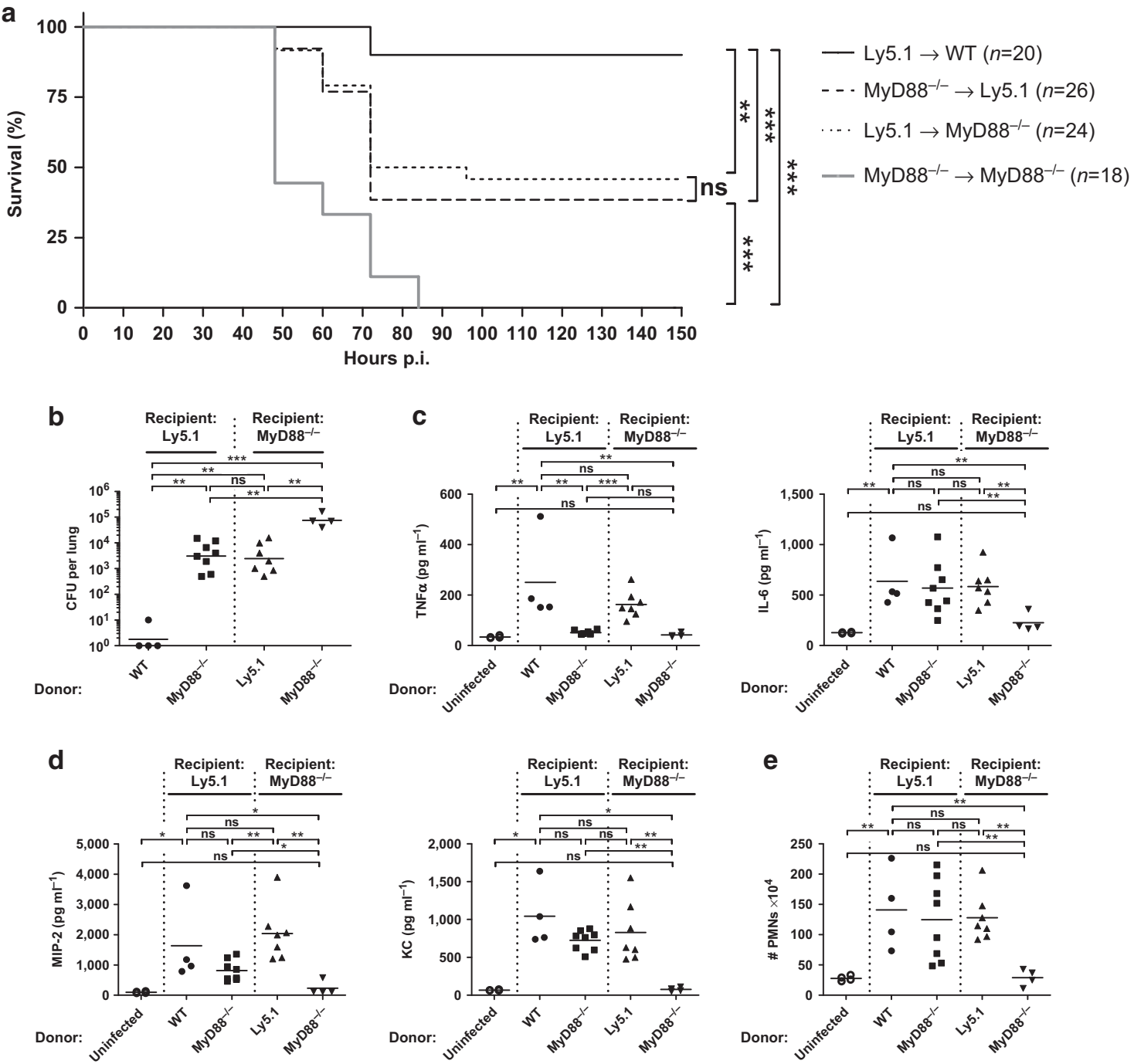

Figure 1 Myeloid differentiation factor 88 (MyD88) signaling in hematopoietic and non-hematopoietic cells is necessary for survival and chemokinedependent neutrophil recruitment. Lethally irradiated recipient Ly5.1 (wild type mice expressing the congenic marker Ly5.1) or MyD88 ${ }^{-1-}$ mice were reconstituted with bone marrow (BM) cells from donor wild type (WT), Ly5.1 or MyD88 ${ }^{-1}-$ mice. After 8 weeks of reconstitution, mice were intranasally inoculated with either a low dose (a) or a high dose (b-e) of Streptococcus pneumoniae. (a) Survival curve of $S$. pneumoniae-infected BM chimeric mice. Data are pooled from four individual experiments with three to eight mice per group. Statistics were calculated using log-rank (Mantel-Cox) test; ** $P<0.01$ and ${ }^{* \star *} P<0.001$. (b) S. pneumoniae burden in total lung homogenates $18 \mathrm{~h}$ post infection (p.i.) (c) Tumor necrosis factor $\alpha$ (TNF $\alpha$ ), interleukin-6 (IL-6), and (d) macrophage inflammatory protein 2 (MIP-2) and keratinocyte chemoattractant (KC) levels in total lung homogenates $18 \mathrm{~h}$ p.i. measured by enzyme-linked immunosorbent assay (ELISA). (e) Fluorescence-activated cell sorting (FACS) analysis of CD11c ${ }^{-} \mathrm{MHCII}^{-} \mathrm{CD}_{11 b^{\text {high }}} \mathrm{Ly} 6 \mathrm{C}^{\text {int }}$ neutrophils (PMNs; excluding CD11 $\mathrm{c}^{-} \mathrm{MHCII}^{-} \mathrm{CD} 11 \mathrm{~b}^{\text {int }} \mathrm{Ly} 6 \mathrm{C}^{\mathrm{hi}}$ monocytes). Shown are total cell numbers in lungs from mice $18 \mathrm{~h}$ p.i. (b-e) Data points depict individual mice. Bars indicate mean values. Data represent two pooled experiments with two to four mice per group. Statistics were calculated using Mann-Whitney test; ${ }^{\star} P<0.05,{ }^{\star \star} P<0.01$, ${ }^{\star * \star} P<0.001$, and ns, not significant.

WT $\rightarrow$ Ly5.1 mice were barely detectable, whereas in

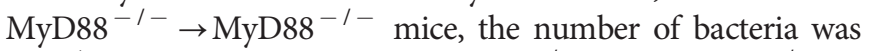
$>10^{4}$ CFUs per lung. Ly5.1 $\rightarrow$ MyD88 $^{-1-}$ and MyD88 $8^{-1-} \rightarrow$ Ly5.1 mice also showed increased CFUs in comparison to the WT group, but significantly less than in MyD88-deficient mice (Figure 1b). Together, these data suggest that MyD88 signaling in both the hematopoietic and non-hematopoietic compartment is required for the control of bacterial replication in the lung.

Since decreased bacterial burden implies an initial functional innate immune response, we next analyzed pulmonary proinflammatory cytokine and chemokine expression. In MyD88-sufficient (WT $\rightarrow$ Ly5.1) mice, S. pneumoniae induced a robust $\mathrm{TNF} \alpha$ and IL-6 release $18 \mathrm{~h}$ p.i. (Figure 1c). TNF $\alpha$ levels were also strongly enhanced in infected Ly5.1 $\rightarrow$ ${\mathrm{MyD} 88^{-1-}}^{-1}$ mice, whereas infected MyD88 $8^{-1-} \rightarrow$ Ly5.1

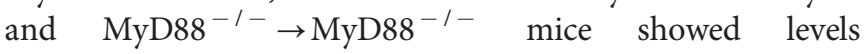
comparable to the uninfected group, indicating that TNF $\alpha$ production is MyD88-dependent and restricted to the hematopoietic compartment, as previously suggested. ${ }^{34,35}$ In contrast, lung IL- 6 and to a lesser extent the PMN-attracting chemokines MIP-2 and KC increased upon infection in mice sufficient for MyD88 signaling in the hematopoietic, the non-hematopoietic, or both compartments, whereas

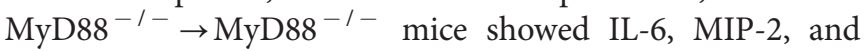


KC levels comparable to uninfected mice (Figure 1c,d). Furthermore, the total number of PMNs in the lungs of Ly5.1 $\rightarrow{\mathrm{MyD} 88^{-/-}}^{-1}$ mice and MyD88 ${ }^{-/-} \rightarrow$ Ly5.1 mice was comparable to $\mathrm{WT} \rightarrow$ Ly5.1, whereas ${\mathrm{MyD} 88^{-1-} \rightarrow}^{-1}$ MyD88 ${ }^{-I-}$ mice completely lacked PMN recruitment (Figure 1e). Thus, MyD88 signaling either in hematopoietic or non-hematopoietic cells is crucial for IL-6, MIP-2, and KC production as well as PMN recruitment to the site of infection.

Together, these data suggest that even though MyD88 signaling in either the hematopoietic or non-hematopoietic compartment improves bacterial clearance, initiates proinflammatory cytokine release and prolongs survival, complete protection against $S$. pneumoniae infection requires MyD88 signaling in both immune and non-immune cells.

\section{MyD88 signaling in CCs partially contributes to early innate responses against $S$. pneumoniae}

We next asked which non-hematopoietic cells contribute to the innate immunity observed in $\mathrm{BM}$ chimeric mice during pneumococcal infection. CCs have been shown to produce proinflammatory cytokines and antimicrobial peptides in response to TLR ligation. ${ }^{21,23,24}$ Therefore, we made use of the MyD88stop (termed MyD88 ${ }^{\mathrm{OFF}}$ ) mouse model, which carries a disruptive stop cassette flanked by two loxP sites between exons 1 and 2 of the Myd88 locus, resulting in a complete loss of MyD88 protein expression. ${ }^{36}$ To assess whether MyD88 signaling in CCs contributes to the aforementioned immune responses, we crossed $\mathrm{MyD} 88^{\mathrm{OFF}}$ mice to mice expressing the Cre recombinase under the control of the CC secretory protein (CCSP) promoter (CCSP-Cre mice). ${ }^{37}$ The resulting offspring (termed CCSP-MyD88 ${ }^{\mathrm{ON}}$ mice) were homozygous for the stop cassette and transgenic for Cre, thus allowing only CCSP ${ }^{+}$cells to induce $M y d 88$ gene expression.

To directly test if MyD88 expression in CCs was sufficient to protect mice from S. pneumoniae infection, WT, CCSP-MyD88 ${ }^{\mathrm{ON}}$, and $\mathrm{MyD} 88^{\mathrm{OFF}}$ mice were infected with a low dose of S. pneumoniae and survival was monitored for 14 days. WT mice showed a survival rate of $80 \%$ whereas all $\mathrm{CCSP}-\mathrm{MyD} 88^{\mathrm{ON}}$ and $\mathrm{MyD} 88^{\mathrm{OFF}}$ mice succumbed to the infection within $96 \mathrm{~h}$ (Figure 2a). We next examined whether the impaired survival of CCSP-MyD88 ${ }^{\mathrm{ON}}$ mice was due to a defect in bacterial clearance. WT, CCSP-MyD $88^{\mathrm{ON}}$, and MyD88 ${ }^{\text {OFF }}$ mice were infected with a high dose of S. pneumoniae, and bacterial burden was analyzed at $18 \mathrm{~h}$ p.i. by determining CFUs in lung homogenates and staining $S$. pneumoniae using immunohistochemistry. WT mice showed drastically reduced CFUs compared to CCSP-MyD $88^{\mathrm{ON}}$ and $\mathrm{MyD} 88^{\mathrm{OFF}}$ mice. However, reactivation of MyD88 signaling in CCSP-MyD8 $8^{\mathrm{ON}}$ mice led to a small but significant reduction in bacterial burden compared to $\mathrm{MyD} 88^{\mathrm{OFF}}$ mice (Figure 2b and Supplementary Figure S3a).

To further elucidate the relevance of MyD88 signaling in CCs upon S. pneumoniae infection, we next analyzed proinflammatory cytokine and chemokine expression. As expected from our results using BM chimeras, S. pneumoniae infection caused a significant increase in TNF $\alpha$ production in WT mice, while in
$\mathrm{CCSP}-\mathrm{MyD} 88^{\mathrm{ON}}$ and $\mathrm{MyD} 88^{\mathrm{OFF}}$ mice, TNF $\alpha$ levels were as low as in uninfected controls (Figure 2c). In contrast, in CCSP-MyD $88^{\mathrm{ON}}$ mice, IL-6, MIP-2, and KC levels although lower than in WT controls were significantly higher than in $\mathrm{MyD} 88^{\mathrm{OFF}}$ mice (Figure 2c,d). Similarly, WT and, to a lesser extent CCSP-MyD88 ${ }^{\mathrm{ON}}$ mice, showed increased PMN numbers in the lung compared to MyD88 ${ }^{\mathrm{OFF}}$ mice (Figure 2e).

Thus, our data suggest that while TNF $\alpha$ production in response to $S$. pneumoniae is strictly dependent on MyD88 expression in hematopoietic cells, IL-6, MIP-2, and KC secretion and thus, recruitment of PMNs to the lungs, is partially dependent on MyD88 expression in CCs.

In addition to the extensive PMN infiltration of the lungs, it has been described that $S$. pneumoniae infection is accompanied by transient immunopathology characterized by a marked inflammatory reaction, disruption of normal airway architecture, local bleeding, and alveolar exudates. ${ }^{31}$ Indeed, pulmonary tissue from WT mice stained with hematoxylin and eosin showed pronounced inflammatory cellular infiltration and local epithelial injury (Figure 2f and Supplementary Figure S4a). In contrast, the lungs of CCSP-MyD88 ${ }^{\mathrm{ON}}$ and $\mathrm{MyD} 88^{\mathrm{OFF}}$ mice displayed significantly reduced pathology scores. In addition, we tested for myeloperoxidase (MPO), a marker of activated PMNs with microbicidal activity. ${ }^{38}$ WT mice, but neither CCSP-MyD $88^{\mathrm{ON}}$ nor $\mathrm{MyD} 88^{\mathrm{OFF}}$ mice, showed extensive $\mathrm{MPO}^{+} \mathrm{PMN}$ infiltration (Figure $2 \mathrm{~g}$ and Supplementary Figure S4b). In summary, although reactivation of $\mathrm{MyD} 88$ signaling in CCs contributes to chemokine expression and PMN recruitment, it is insufficient to provide complete protection against pneumococcal infection.

\section{CCs produce MIP-2, IL-6, and SP-D in a MyD88-dependent manner during pneumococcal infection}

To determine whether intrinsic MyD88 signaling in CCs directly triggers the production of IL- 6 and MIP- 2 by these cells, or whether indirect effects on hematopoietic cells are responsible for the production of these inflammatory mediators, we made use of CCSP-Cre mice crossed to red fluorescent protein (RFP) reporter mice, thereby terminally labeling $\mathrm{CCSP}^{+}$cells with RFP expression driven by the ubiquitous ROSA26 promoter. ${ }^{39}$ Lung sections from CCSP-RFP mice showed RFP signals only within cells located in the bronchiolar airways confirming specific RFP expression in CCs (Figure 3a). Subsequently, we infected CCSP-RFP mice and sorted $\mathrm{RFP}^{+} \mathrm{CCs} 18 \mathrm{~h}$ p.i. $\mathrm{RFP}^{+}$CCs from infected and uninfected CCSP-RFP mice showed equally low Tnfa levels, whereas increased $I l 6$ and Mip2 transcript levels were detected in CCs from infected CCSP-RFP mice compared to uninfected controls (Figure 3b). We next sorted AMs and PMNs from infected WT, CCSP-MyD88 ${ }^{\mathrm{ON}}$, and $\mathrm{MyD} 88^{\mathrm{OFF}}$ mice, and measured Tnfa, Il6, and Mip2 gene expression by quantitative PCR. In WT mice, Tnfa and Mip2 transcript levels were detectable in both AMs and PMNs, whereas Il6 was only detectable in AMs. Furthermore, expression of all the three genes in both AMs and PMNs were equally reduced in CCSP-MyD88 ${ }^{\mathrm{ON}}$ and $\mathrm{MyD} 88^{\mathrm{OFF}}$ compared to WT-derived cells. Of note, $M y D 88$ expression was neither detectable in AMs 

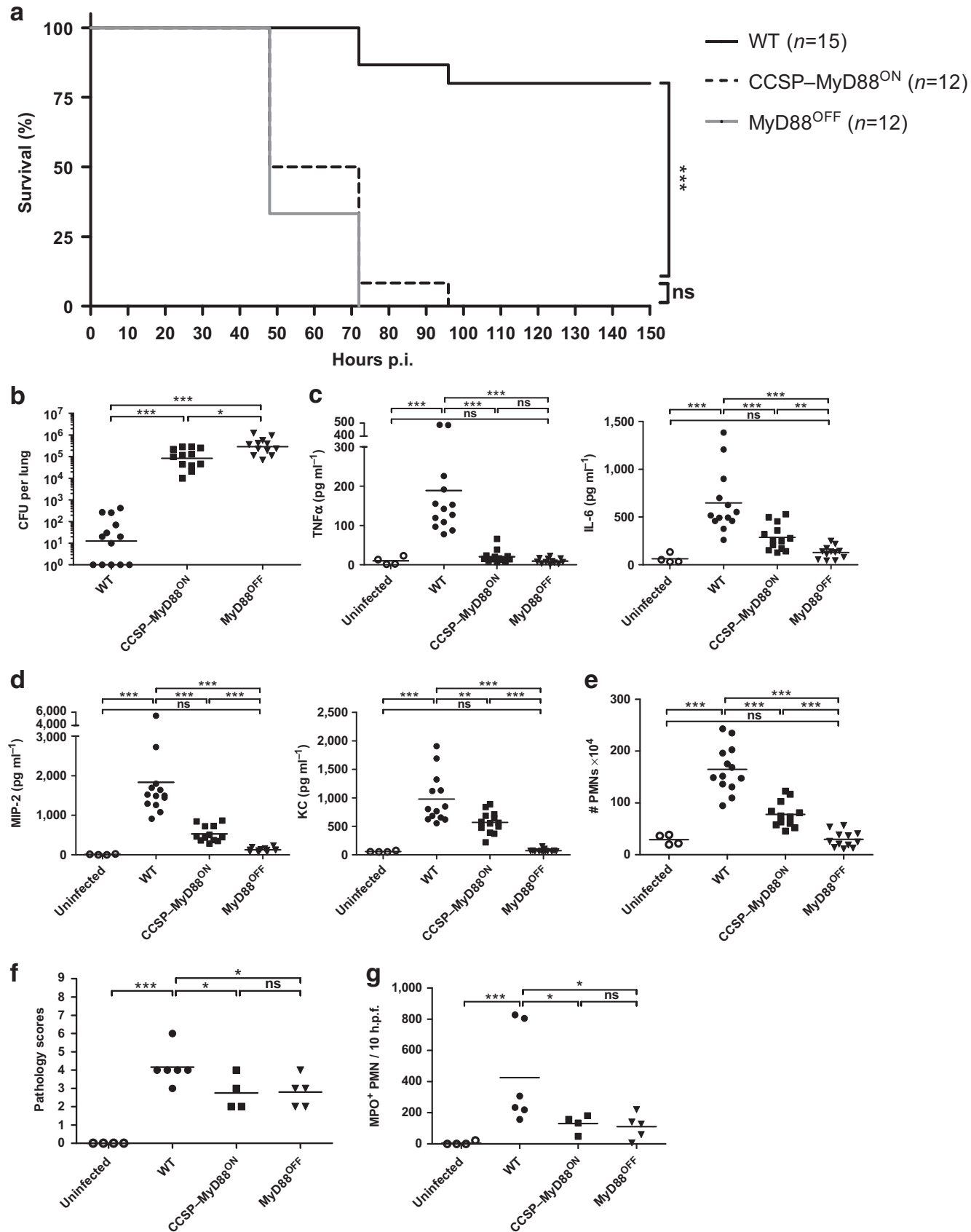

Figure 2 Myeloid differentiation factor 88 (MyD88) signaling in club cells contributes to proinflammatory cytokine production and control of bacterial burden. Wild type (WT), club cell secretory protein (CCSP)-MyD88 ${ }^{\mathrm{ON}}$ and MyD88 ${ }^{\mathrm{OFF}}$ mice were intranasally inoculated with either a low dose (a) or a high dose (b-g) of Streptococcus pneumoniae. (a) Survival curve of $\mathbf{S}$. pneumoniae-infected mice. Data show two pooled individual experiments with five to nine mice per group. Statistics were calculated using log-rank (Mantel-Cox) test; ${ }^{* \star \star} P<0.001$ and ns, not significant. (b) $S$. pneumoniae burden in total lung homogenates $18 \mathrm{~h}$ post infection (p.i.). (c) Tumor necrosis factor $\alpha$ (TNF $\alpha$ ), interleukin-6 (IL-6), and (d) macrophage inflammatory protein 2 (MIP-2) and keratinocyte chemoattractant (KC) levels in total lung homogenates $18 \mathrm{~h}$ p.i. measured by enzyme-linked immunosorbent assay (ELISA). (e) Fluorescenceactivated cell sorting (FACS) analysis of CD11 ${ }^{-}$MHCII $^{-}$CD11 $b^{\text {high }}$ Ly6 $C^{\text {int }}$ neutrophils (PMNs; excluding CD11c ${ }^{-}$MHCII $^{-} C^{-}$11 $b^{\text {int }}$ Ly6C ${ }^{\text {hi }}$ monocytes). Shown are total cell numbers in lungs from WT, CCSP-MyD88 ${ }^{\mathrm{ON}}$, and MyD88 ${ }^{\mathrm{OFF}}$ mice $18 \mathrm{~h}$ p.i. Evaluation of (f) hematoxylin and eosin (H\&E) and (g) myeloperoxidase (MPO) staining of lung tissue from uninfected and $S$. pneumoniae-infected mice $18 \mathrm{~h}$ p.i. Tissue sections were evaluated in a blinded manner. Dot plots depict (f) histopathological scoring and (g) MPO ${ }^{+}$PMNs counted within 10 high power fields (h.p.f.). (b-e) Data represent three pooled individual experiments with three to five mice per group. $(\mathbf{f}, \mathbf{g})$ Data are pooled from two individual experiments with two to three mice per group. (b-g) Data points depict individual mice. Bars indicate mean value. Statistics were calculated using Mann-Whitney test; ${ }^{*} P<0.05,{ }^{* \star} P<0.01$, and ${ }^{* * *} P<0.001$.

or PMNs from CCSP-MyD88 ${ }^{\mathrm{ON}}$ nor in $\mathrm{MyD} 88^{\mathrm{OFF}}$ mice (Supplementary Figure S5). Thus, upon S. pneumoniae infection CCs produce IL-6 and MIP-2 in a MyD88dependent cell-intrinsic manner.
Finally, we asked whether the decreased bacterial burden seen in CCSP-MyD88 ${ }^{\mathrm{ON}}$ mice (Figure 2b) might be attributed to antimicrobial effects induced by CCs. The antimicrobial peptide SP-D can bind to several microbes including 
a

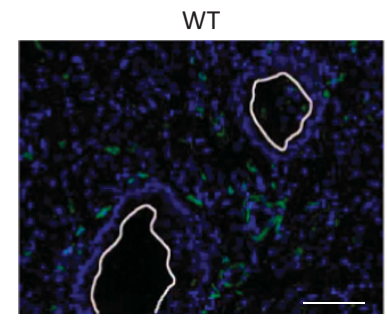

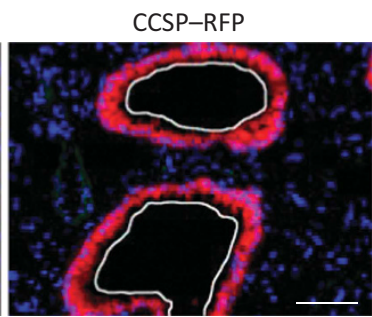

b

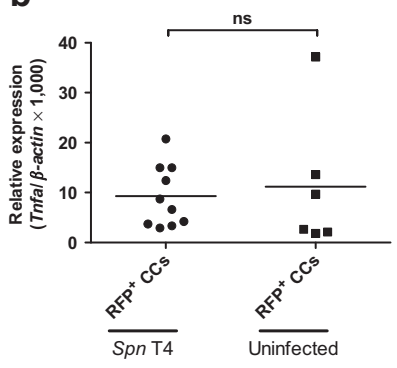

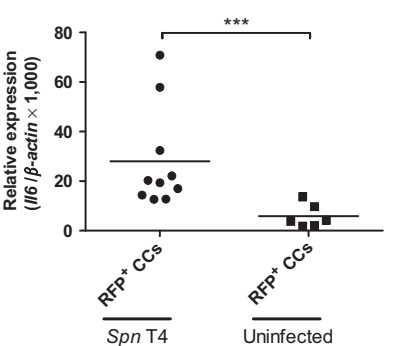
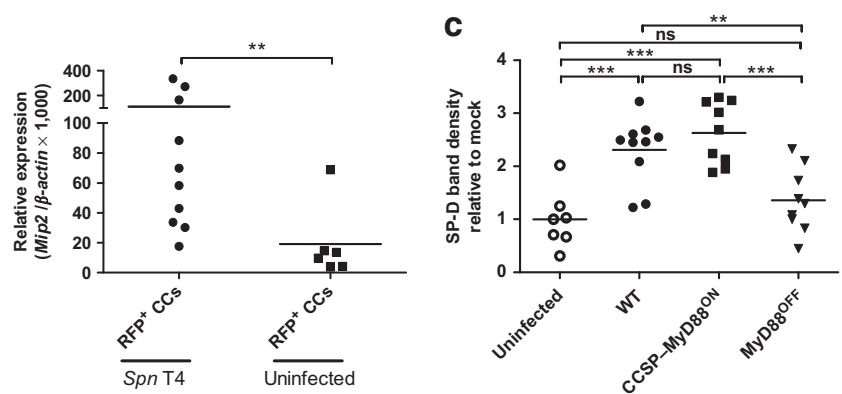

Figure 3 Club cells produce proinflammatory cytokines and surfactant protein D (SP-D) dependent on myeloid differentiation factor 88 (MyD88) signaling. (a) Cryosections from lungs of naive wild type (WT) and club cell secretory protein-red fluorescent protein (CCSP-RFP) mice were stained with anti-RFP antibody. Representative immunofluorescence pictures from one mouse of each group are shown. Bar $=50 \mu \mathrm{m}$. (b) CCSP-RFP mice were either intranasally inoculated with a high dose of Streptococcus pneumoniae (Spn T4) or treated with phosphate-buffered saline (PBS, uninfected). $\mathrm{CCSP}-R F P^{+}$cells were fluorescence-activated cell sorting-(FACS) sorted and analyzed for Tnfa, I/6, and Mip2 gene expression levels by quantitative $\mathrm{PCR}$. Gene expression is shown relative to $\beta$-actin expression. (c) WT, CCSP-MyD88 ${ }^{\mathrm{ON}}$, and MyD88 ${ }^{\mathrm{OFF}}$ mice were intranasally inoculated with a high dose of $S$. pneumoniae and bronchoalveolar lavage (BAL) was taken from uninfected and infected mice $18 \mathrm{~h}$ post infection (p.i.) BAL proteins were analyzed by western blot for SP-D content. SP-D band densities were quantified with ImageJ gel analyzer and depicted relative to the mean value of SP-D levels of uninfected mice. (a) Data show one mouse out of two mice from each group from one experiment. (b) Data are pooled from three individual experiments with two to four mice per group. (c) Data represent three pooled individual experiments with one to four mice per group. (b,c) Data points depict individual mice. Bars indicate mean values. Statistics were calculated using Mann-Whitney test; ${ }^{* *} P<0.01,{ }^{* * *} P<0.001$, and ns, not significant.

S. pneumoniae leading to enhanced phagocytosis of bacteria by PMNs. ${ }^{40}$ In addition, SP-D-deficient mice show an increased susceptibility to colonization and infection with $S$. pneumoniae even though recruitment of PMNs is not altered. ${ }^{41}$ Therefore, we analyzed SP-D levels within bronchoalveolar lavage (BAL) of infected and uninfected mice. Remarkably, WT and CCSP-MyD $88^{\mathrm{ON}}$ mice had equally increased SP-D levels, whereas $\mathrm{MyD} 88^{\mathrm{OFF}}$ mice showed no increase in SP-D compared to uninfected controls (Figure $3 \mathrm{c}$ and Supplementary Figure S6a).

Altogether, our data indicate that during $S$. pneumoniae infection intrinsic MyD88 signaling in CCs is required for IL-6, MIP-2, and SP-D production, but not for TNF $\alpha$ secretion.

\section{MyD88 signaling in $\mathrm{CD}^{11 \mathrm{c}^{+}}$and $\mathrm{LysM}^{+}$cells is required for early innate responses, but does not prevent bacterial dissemination}

MyD88 signaling in lung epithelial CCs led to proinflammatory cytokine secretion and restored SP-D production. However, $\mathrm{CCSP}-\mathrm{MyD} 88^{\mathrm{ON}}$ mice succumbed to pneumococcal infection due to uncontrolled bacterial outgrowth. Early bacterial clearance is one major function of lung-resident hematopoietic cells, such as AMs and DCs, followed by PMNs. To define the role of MyD88 signaling in these cell populations, we crossed $\mathrm{MyD}_{88^{\mathrm{OFF}}}$ mice to CD11c-Cre mice ${ }^{42}$ or LysM-Cre mice ${ }^{43}$ (expressing Cre recombinase under the control of Cd11c (Itgax) or the LysM promoter, respectively), only the offspring homozygous for $\mathrm{MyD} 88^{\mathrm{OFF}}$ and transgenic for Cre (termed
$\mathrm{CD} 11 \mathrm{c}-\mathrm{MyD}^{\mathrm{ON}}$ and $\mathrm{LysM}-\mathrm{MyD} 88^{\mathrm{ON}}$ mice) will have functional $\mathrm{Myd} 88$ gene expression in $\mathrm{CD} 11 \mathrm{c}^{+}$or $\mathrm{LysM}^{+}$cells. Because CD11c is considered as a crucial marker for DCs within the lungs but is also expressed in AMs, ${ }^{44}$ and LysM was shown to be expressed predominantly in AMs and $\mathrm{PMNs}^{43}$ we first evaluated the targeting efficiency in the lungs using $\mathrm{CD} 11 \mathrm{c}-\mathrm{Cre}-$ and LysM-Cre-RFP reporter mice. In CD11c-RFP mice, $80 \%$ of $\mathrm{AMs}, 70 \%$ of DCs, and $<5 \%$ of PMNs were $\mathrm{RFP}^{+}$. In contrast, LysM-RFP mice targeted 75\% of AMs and only $20 \%$ of PMNs and DCs (Supplementary Figure S7a and b). Lung histology of CD11c-RFP and LysM-RFP mice showed RFP signals only within cells located in pulmonary parenchyma, thus confirming RFP expression exclusively in hematopoietic-derived cells (Supplementary Figure S7c).

To test whether MyD88 reactivation in $\mathrm{CD}_{11 c^{+}}$or LysM $^{+}$cells is sufficient for survival, we infected WT, $\mathrm{CD} 11 \mathrm{c}-\mathrm{MyD}_{8}{ }^{\mathrm{ON}}{ }^{\mathrm{LysM}}-\mathrm{MyD} 88^{\mathrm{ON}}$, and $\mathrm{MyD} 88^{\mathrm{OFF}}$ mice with a low dose of S. pneumoniae TIGR4, and assessed survival over a period of 14 days. WT mice showed a survival rate of $80 \%$, whereas all MyD88 ${ }^{\mathrm{OFF}}$ mice succumbed to infection by $72 \mathrm{~h}$. In contrast, $\sim 25 \%$ of $\mathrm{CD} 11 \mathrm{c}-\mathrm{MyD} 88^{\mathrm{ON}}$ and LysM$\mathrm{MyD} 88^{\mathrm{ON}}$ mice survived the infection, suggesting that MyD88 reactivation in either $\mathrm{CD}_{11 \mathrm{c}^{+}}$or $\mathrm{LysM}^{+}$cells is partially required to control the infection. Of note, mice from the WT, $\mathrm{CD} 11 \mathrm{c}-\mathrm{MyD} 88^{\mathrm{ON}}$, or LysM-MyD88 ${ }^{\mathrm{ON}}$ group succumbed to the infection only after $72 \mathrm{~h}$, implying that MyD88 signaling in these cell populations is crucial in the early phase of infection 
a

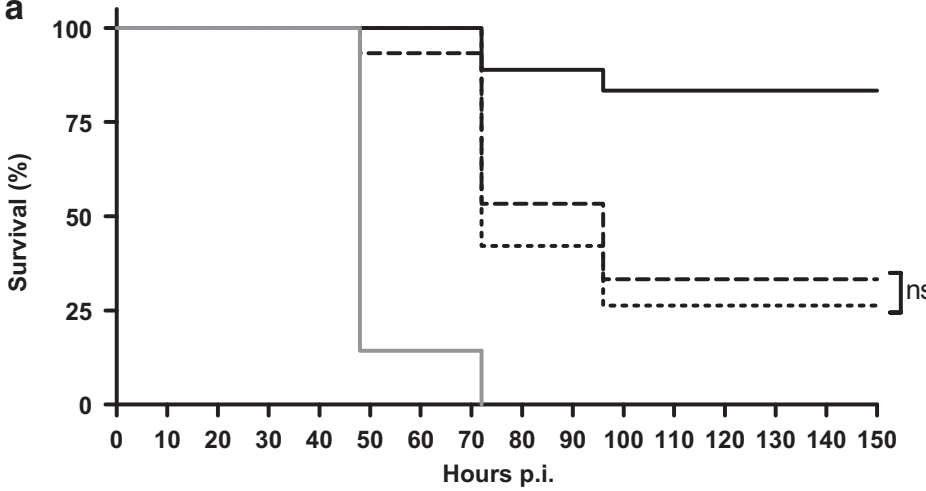

b

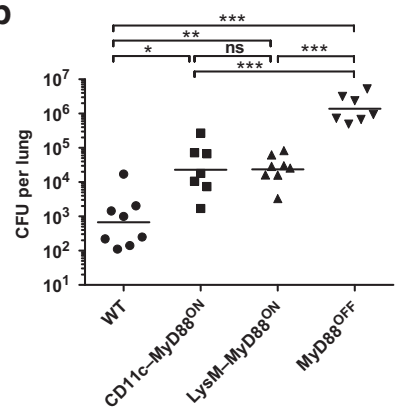

d

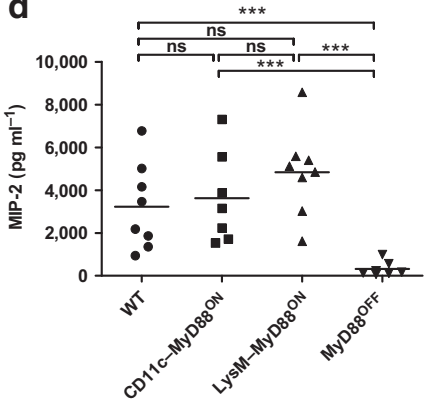

f

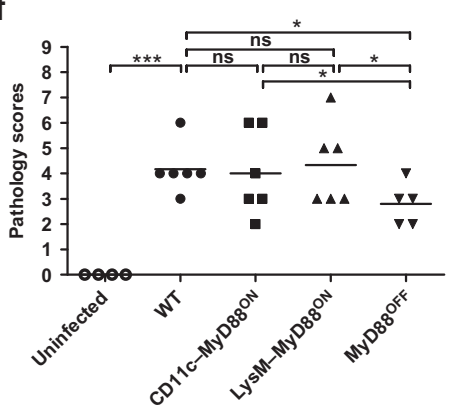

C
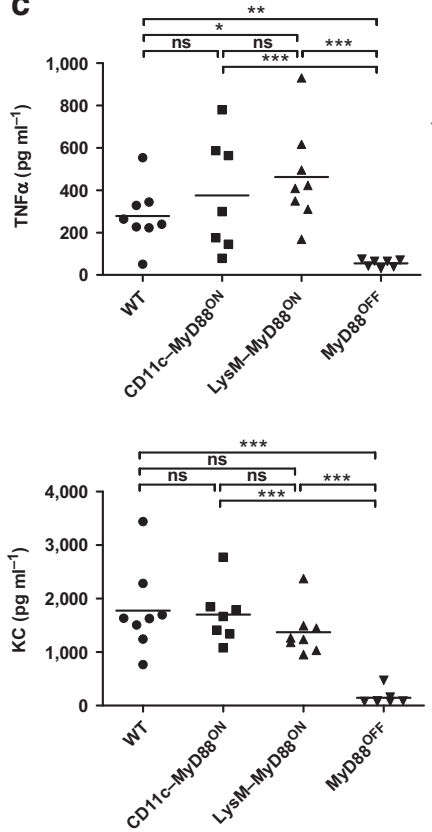

g

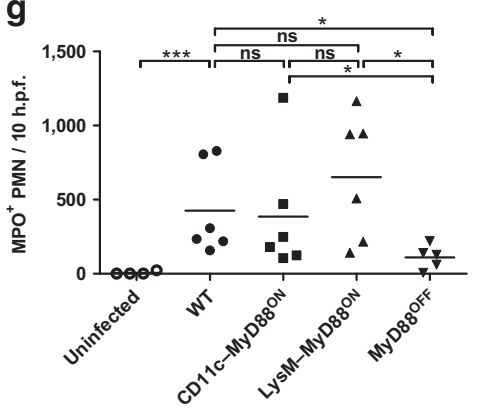

e
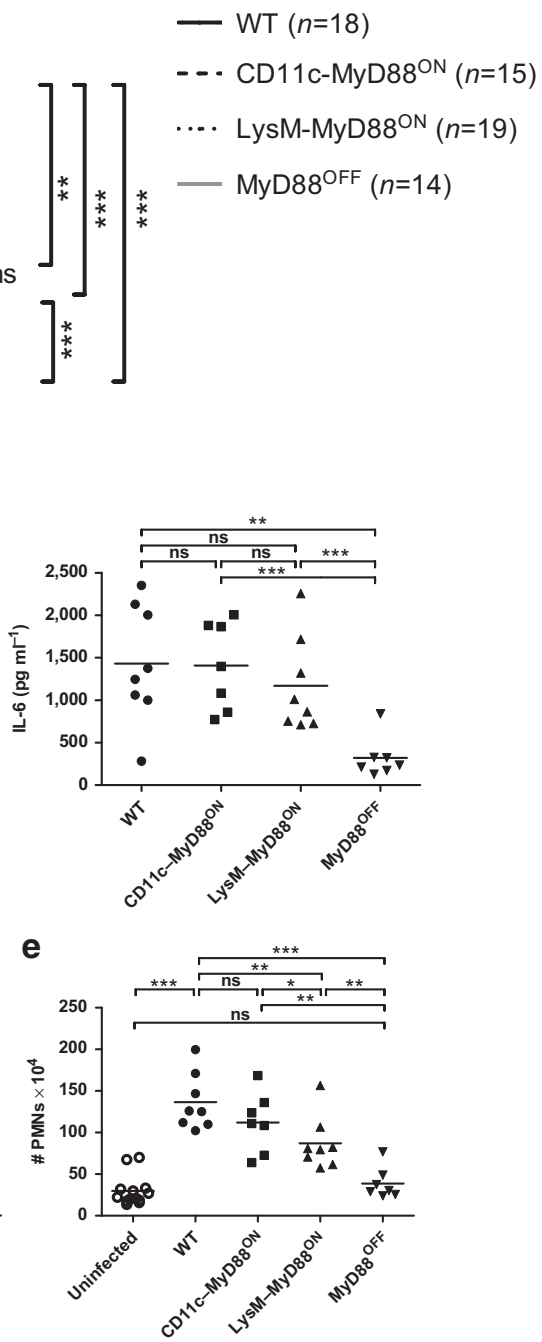

h

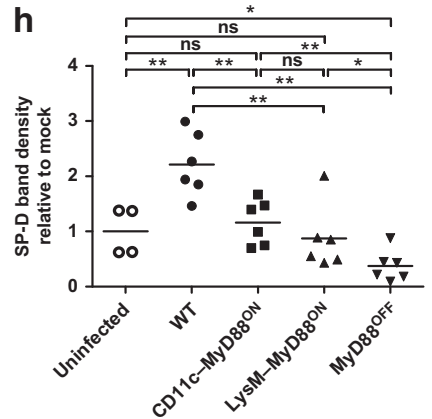

Figure 4 Myeloid differentiation factor 88 (MyD88) reactivation in CD11c or lysozyme M (LySM)-positive cells initiates early innate immune responses leading to improved survival and reduced bacterial burden. Wild type (WT), CD11c-MyD88 ${ }^{\mathrm{N}}$, LysM-MyD88 ${ }^{\mathrm{ON}}$, and MyD88 ${ }^{\mathrm{OFF}}$ mice were intranasally inoculated with either a low dose (a) or a high dose (b-h) of Streptococcus pneumoniae. (a) Survival curve of S. pneumoniae-infected mice. Data show two pooled individual experiments with 6-10 mice per group. Statistics were calculated using log-rank (Mantel-Cox) test; ${ }^{* *} P<0.01$ and ${ }^{* * *} P<0.001$. (b) $S$. pneumoniae burden in total lung homogenates $18 \mathrm{~h}$ post infection (p.i.). (c) Tumor necrosis factor $\alpha$ (TNF $\alpha$ ), interleukin-6 (IL-6), and (d) macrophage inflammatory protein 2 (MIP-2) and keratinocyte chemoattractant (KC) levels in total lung homogenates $18 \mathrm{~h}$ p.i. measured by enzyme-linked immunosorbent assay (ELISA). (e) Fluorescence-activated cell sorting (FACS) analysis of CD11C ${ }^{-}$MHCII $^{-}$CD11b $^{\text {high }}$ Ly6C $^{\text {int }}$ neutrophils (PMNs; excluding $\mathrm{CD} 11 \mathrm{c}^{-} \mathrm{MHCII}^{-} \mathrm{CD} 11 \mathrm{~b}^{\text {int }} \mathrm{Ly} 6 \mathrm{C}^{\text {hi }}$ monocytes). Shown are total cell numbers in lungs from mice $18 \mathrm{~h}$ p.i. Uninfected group represents pooled data from three to four phosphate-buffered saline (PBS)-treated mice from each mouse line: WT, CD11c-MyD88 ${ }^{\mathrm{ON}}$, LysM-MyD88 ${ }^{\mathrm{ON}}$, and MyD88 ${ }^{\mathrm{OFF}}$.

Evaluation of (f) hematoxylin and eosin (H\&E) and (g) myeloperoxidase (MPO) staining of lung tissue from uninfected and S. pneumoniae-infected mice $18 \mathrm{~h}$ p.i. Tissue sections were evaluated in a blinded manner. Dot plots depict (f) histopathological scoring and (g) MPO ${ }^{+} \mathrm{PMNs}$ counted within 10 high power fields (h.p.f.). (h) Bronchoalveolar lavage (BAL) was taken from uninfected and infected mice $18 \mathrm{~h}$ p.i. BAL proteins were analyzed by western blot for surfactant protein D (SP-D) content. SP-D band densities were quantified with ImageJ gel analyzer and depicted relative to the mean value of SP-D levels of uninfected mice. (b-e) Data represent two pooled individual experiments with three to four mice per group. $(\mathbf{f}, \mathbf{g})$ Data are pooled from two individual experiments with two to four mice per group. (h) Data show two pooled individual experiments with one to three mice per group. (b-h) Data points depict individual mice. Bars indicate mean values. Statistics were calculated using Mann-Whitney test; ${ }^{*} P<0.05,{ }^{* \star} P<0.01$, ${ }^{\star * \star} P<0.001$, and ns, not significant. 
(Figure 4a). Indeed, the bioluminescence in CD11c$\mathrm{MyD}_{88^{\mathrm{ON}}}$, LysM-MyD88 ${ }^{\mathrm{ON}}$, and $\mathrm{MyD} 88^{\mathrm{OFF}}$ mice, infected with a low dose of TIGR4X, was significantly higher than in WT mice until $28 \mathrm{~h}$ p.i. However, the signal decreased by $43 \mathrm{~h}$ within $\mathrm{CD} 11 \mathrm{c}-\mathrm{MyD} 88^{\mathrm{ON}}$ and LysM-MyD88 ${ }^{\mathrm{ON}}$ mice in comparison to $\mathrm{MyD} 88^{\mathrm{OFF}}$ mice, demonstrating that MyD88 signaling in $\mathrm{CD}_{11 \mathrm{c}^{+}}$or $\mathrm{LysM}^{+}$cells is important for the early containment of bacterial outgrowth (Supplementary Figure S8a). Computed tomography scanning at $67 \mathrm{~h}$ p.i. showed that $S$. pneumoniae disseminated across the whole lungs in $\mathrm{CD} 11 \mathrm{c}-\mathrm{MyD} 88^{\mathrm{ON}}$, LysM-MyD88 ${ }^{\mathrm{ON}}$, and $\mathrm{MyD} 88^{\mathrm{OFF}}$ mice (Supplementary Figure S8b).

To further investigate the MyD88-dependent contribution of $\mathrm{CD}_{11 \mathrm{c}^{+}}$and $\mathrm{LysM}^{+}$cells in the early phase of infection, we infected mice with a high dose of $S$. pneumoniae. Consistent with the IVIS data, bacterial burden $18 \mathrm{~h}$ p.i. was significantly reduced in the lungs of WT mice compared to $\mathrm{CD} 11 \mathrm{c}-\mathrm{MyD}_{8}{ }^{\mathrm{ON}}$, LysM-MyD88 ${ }^{\mathrm{ON}}$, and $\mathrm{MyD} 88^{\mathrm{OFF}}$ mice. However, CD11c-MyD88 ${ }^{\mathrm{ON}}$ and LysM-MyD88 ${ }^{\mathrm{ON}}$ mice showed significantly less CFUs than $\mathrm{MyD} 88^{\mathrm{OFF}}$ mice, demonstrating that $\mathrm{MyD} 88$ signaling in $\mathrm{CD}_{11 \mathrm{c}^{+}}$and $\mathrm{LysM}^{+}$cells partially controls early bacterial burden (Figure $\mathbf{4 b}$ and Supplementary Figure S3b).

Reactivation of MyD88 signaling in $\mathrm{CD}_{11 c^{+}}$or LysM $^{+}$cells was sufficient to restore total lung TNF $\alpha$, IL-6, MIP-2, and KC to WT levels, whereas MyD88 ${ }^{\mathrm{OFF}}$ mice produced significantly less inflammatory cytokines (Figure 4c,d). Moreover, sorted AMs from CD11c-MyD88 ${ }^{\mathrm{ON}}$ and $\mathrm{LysM}-\mathrm{MyD} 88^{\mathrm{ON}}$ mice expressed Myd88, Tnfa, Mip2, and Il6 transcript levels comparable or even higher than WT cells and significantly increased in comparison to MyD88 ${ }^{\mathrm{OFF}}$ mice (Supplementary Figure S9a). In turn, the levels of Myd88, Tnfa, and Mip2 in the PMN population were similar between LysM-MyD88 ${ }^{\mathrm{ON}}$ and WT mice, but reduced in the $\mathrm{CD} 11 \mathrm{c}-\mathrm{MyD} 88^{\mathrm{ON}}$ group. However, WT, CD11c-MyD88 ${ }^{\mathrm{ON}}$, and, to a lesser extent, LysM-MyD88 ${ }^{\mathrm{ON}}$ mice showed significantly more PMNs than MyD88 ${ }^{\text {OFF }}$ mice, demonstrating that MyD88 signaling in $\mathrm{CD}_{11 \mathrm{c}^{+}}$or $\mathrm{LysM}^{+}$cells is sufficient for the initiation of PMN recruitment to the site of infection (Figure 4e). Hematoxylin and eosin staining of WT, CD11c-MyD88 ${ }^{\mathrm{ON}}$, and LysM-MyD88 ${ }^{\mathrm{ON}}$ pulmonary tissue showed a pronounced inflammatory cellular infiltration and local epithelial injury, whereas the lungs of $\mathrm{MyD} 88^{\mathrm{OFF}}$ mice showed significantly reduced pathology scores in comparison to the aforementioned groups (Figure 4f and Supplementary Figure S4c). In addition, WT, CD11c-MyD88 ${ }^{\mathrm{ON}}$, and LysM-MyD88 ${ }^{\mathrm{ON}}$ mice showed an extensive $\mathrm{MPO}^{+}{ }^{+} \mathrm{PMN}$ infiltration in comparison to MyD88 ${ }^{\mathrm{OFF}}$ mice (Figure 4g and Supplementary Figure S4d). Thus, MyD88 reactivation in $\mathrm{CD}_{11 c^{+}}$and $\mathrm{LysM}^{+}$cells is sufficient to restore early innate responses, but not for complete bacterial control.

Apoptosis of AMs has been proposed as a crucial mechanism contributing to the clearance of pneumococcal infection. ${ }^{45}$ Inflammatory PMNs participate in this process by increasing TNF-related apoptosis-inducing ligand (TRAIL) expression, thus leading to caspase- 3 activation in AMs. ${ }^{45}$ Consequently, we evaluated whether this apoptotic pathway was related to the impaired bacterial control observed in CD11c-MyD88 ${ }^{\mathrm{ON}}$ and LysM-MyD88 ${ }^{\mathrm{ON}}$ mice. However, WT, CD11c-MyD88 ${ }^{\mathrm{ON}}$, and LysM-MyD88 ${ }^{\mathrm{ON}}$ mice had significantly reduced AM numbers in comparison to MyD88 ${ }^{\mathrm{OFF}}$ mice (Supplementary Figure S9b). Moreover, AMs and PMNs isolated from WT, $\mathrm{CD} 11 \mathrm{c}-\mathrm{MyD} 88^{\mathrm{ON}}$, and $\mathrm{LysM}-\mathrm{MyD} 88^{\mathrm{ON}}$ mice, showed significantly higher caspase-3 and Trail transcript levels, respectively, than $\mathrm{MyD} 88^{\mathrm{OFF}}$ cells (Supplementary Figure S9a), suggesting that this mechanism is intact in $\mathrm{CD} 11 \mathrm{c}-\mathrm{MyD} 88^{\mathrm{ON}}$ and LysM-MyD88 ${ }^{\mathrm{ON}}$ mice.

In contrast to phagocytic AMs and PMNs, DCs have less antibacterial activity and rather link the innate and adaptive immune system by presenting processed antigens from the lung lumen to T-cells in the peripheral LNs. Particularly, CD103 ${ }^{+}$ DCs migrate to lung-draining LNs upon antigen encounter. ${ }^{46}$ Hence, we analyzed if cell-specific reactivation of MyD88 signaling caused alterations in $\mathrm{CD}_{103}{ }^{+}$DC numbers in the lungs from infected mice. WT, CD11c-MyD88 ${ }^{\mathrm{ON}}$, and LysM-MyD88 ${ }^{\mathrm{ON}}$ mice had significantly decreased CD103 ${ }^{+}$ $\mathrm{DC}$ numbers in comparison to $\mathrm{MyD} 88^{\mathrm{OFF}}$ mice (Supplementary Figure S10a). Since migration of DCs to lung-draining LNs was shown to be CCR7-dependent, ${ }^{46}$ we tested for $\mathrm{C} c r 7$ gene expression in sorted pulmonary $\mathrm{CD}_{103}{ }^{+}$DCs from infected mice. CD103 ${ }^{+}$DCs from WT, $\mathrm{CD} 11 \mathrm{c}-\mathrm{MyD} 8^{\mathrm{ON}}$, and LysM-MyD88 ${ }^{\mathrm{ON}}$ mice showed significantly increased $\mathrm{Ccr} 7$ transcript levels in comparison to $\mathrm{CD}_{103}{ }^{+} \mathrm{DCs}$ from $\mathrm{MyD} 88^{\mathrm{OFF}}$ mice (Supplementary Figure S10b). Thus, functional MyD88 signaling in PMNs, AMs, and $\mathrm{CD}_{103}{ }^{+}$DCs restored innate immune responses comparable to $\mathrm{WT}$, but $\mathrm{CD} 11 \mathrm{c}-\mathrm{MyD} 88^{\mathrm{ON}}$ and LysM-MyD88 ${ }^{\mathrm{ON}}$ mice still showed elevated CFU numbers. Therefore, we measured SP-D levels in the BAL of $\mathrm{CD} 11 \mathrm{c}-\mathrm{MyD} 88^{\mathrm{ON}}$ and LysM-MyD88 ${ }^{\mathrm{ON}}$ mice. WT mice had significantly increased SP-D levels, whereas $\mathrm{CD} 11 \mathrm{c}-\mathrm{MyD}_{8} 8^{\mathrm{ON}}$, LysM-MyD88 ${ }^{\mathrm{ON}}$, and $\mathrm{MyD} 88^{\mathrm{OFF}}$ mice showed SP-D levels comparable to uninfected mice, providing an explanation for higher pneumococcal outgrowth in these mice (Figure $4 \mathrm{~h}$ and Supplementary Figure S6b).

In conclusion, reactivation of MyD88 signaling in $\mathrm{CD} 11 \mathrm{c}^{+}$ or LysM ${ }^{+}$cells is sufficient to restore proinflammatory cytokine responses and to recruit PMNs to the site of infection. Likewise, MyD88 signaling is required for the induction of AM apoptosis by initiating Trail and caspase- 3 induction. However, $\mathrm{CD} 11 \mathrm{c}-\mathrm{MyD} 88^{\mathrm{ON}}$ and $\mathrm{LysM}-\mathrm{MyD} 88^{\mathrm{ON}}$ mice have an impaired pneumococcal clearance leading to delayed bacterial outgrowth and increased mortality, which might be at least in part due to diminished upregulation of epithelial-derived antimicrobial peptides.

\section{Lung epithelium, AMs, and PMNs coordinate innate immunity via MyD88 signaling to control pneumococcal infection}

Cell-specific reactivation of MyD88 signaling in AMs, PMNs, or CCs contributes to early innate immune responses by the release of proinflammatory cytokines and chemokines and 
antimicrobial peptides. However, the immune response from either the hematopoietic or lung epithelial compartment alone is not sufficient to rescue mice from S. pneumoniae infection. To test if MyD88 signals derived from the lung epithelium and hematopoietic cells act synergistically to resolve S. pneumoniae infection, we again generated BM chimeric mice. Here, lethally irradiated WT mice were reconstituted with $\mathrm{BM}$ from donor $\mathrm{CD} 11 \mathrm{c}-\mathrm{MyD} 88^{\mathrm{ON}}$, $\mathrm{LysM}-\mathrm{MyD} 88^{\mathrm{ON}}$, or $\mathrm{MyD} 88^{\mathrm{OFF}}$ mice. We also included control chimeras consisting of WT $\rightarrow \mathrm{WT}$ and $\mathrm{MyD} 88^{\mathrm{OFF}} \rightarrow \mathrm{MyD} 8^{\mathrm{OFF}}$ mice. To confirm the early proinflammatory cytokine response and PMN recruitment seen in mouse lines described earlier in this study, we infected reconstituted mice with a high dose of $S$. pneumoniae and analyzed $18 \mathrm{~h}$ p.i. for TNF $\alpha$, IL-6, MIP-2, and KC levels. Our results showed that in $\mathrm{CD} 11 \mathrm{c}-\mathrm{MyD} 88^{\mathrm{ON}} \rightarrow \mathrm{WT}$ and LysM-MyD88 ${ }^{\mathrm{ON}} \rightarrow \mathrm{WT}$ mice no significant differences in cytokine levels were observed in comparison to WT $\rightarrow \mathrm{WT}$ mice, whereas $\mathrm{MyD} 88^{\mathrm{OFF}} \rightarrow \mathrm{WT}$ and $\mathrm{MyD}^{\mathrm{OFF}} \rightarrow \mathrm{MyD}^{\mathrm{O}} 8^{\mathrm{OFF}}$ mice had significantly decreased cytokine levels compared to the other groups (Figure 5a,b). Moreover, PMN and AM numbers in $\mathrm{WT} \rightarrow \mathrm{WT}$ mice were comparable to $\mathrm{CD} 11 \mathrm{c}-\mathrm{MyD} 88^{\mathrm{ON}} \rightarrow$ $\mathrm{WT}$ and LysM-MyD88 ${ }^{\mathrm{ON}} \rightarrow \mathrm{WT}$ mice. In contrast, correlating with the decreased chemokine levels $\mathrm{MyD} 88^{\mathrm{OFF}} \rightarrow \mathrm{WT}$ mice had decreased PMN numbers in comparison to WT $\rightarrow$ WT mice (Figure 5c).

Since MyD88-dependent antimicrobial peptide production in lung epithelial CCs critically contributed to S. pneumoniae containment, we analyzed lung bacterial burdens in BM chimeric mice. CFUs in $\mathrm{WT} \rightarrow \mathrm{WT}$ mice were comparable between $\mathrm{CD} 11 \mathrm{c}-\mathrm{MyD} 88^{\mathrm{ON}} \rightarrow \mathrm{WT}$ and $\mathrm{LysM}-\mathrm{MyD} 88^{\mathrm{ON}} \rightarrow$ WT mice. Contrary to this, $\mathrm{MyD} 88^{\mathrm{OFF}} \rightarrow \mathrm{WT}$ mice had strongly increased CFUs in comparison to $\mathrm{WT} \rightarrow \mathrm{WT}$ mice but still significantly less bacterial burden than $\mathrm{MyD} 88^{\mathrm{OFF}} \rightarrow$ $\mathrm{MyD} 88^{\mathrm{OFF}}$ mice (Figure 5d). Therefore, MyD88 signaling in lung epithelial cells, AMs and PMNs is required to efficiently eradicate bacteria.

Furthermore, we infected the mice with a low dose of S. pneumoniae and monitored their survival over 14 days. Consistent with the results obtained in Figure 1a, all $\mathrm{MyD}_{8}{ }^{\mathrm{OFF}} \rightarrow \mathrm{MyD}^{\mathrm{OFF}}$ mice succumbed to infection, whereas $90 \%$ and $36 \%$ of $\mathrm{WT} \rightarrow \mathrm{WT}$ and $\mathrm{MyD} 88^{\mathrm{OFF}} \rightarrow \mathrm{WT}$ mice survived the infection, respectively. In line with efficient reduction of CFUs, $73 \%$ of $\mathrm{CD} 11 \mathrm{c}-\mathrm{MyD} 88^{\mathrm{ON}} \rightarrow \mathrm{WT}$ mice and $82 \%$ of LysM-MyD88 ${ }^{\mathrm{ON}} \rightarrow$ WT mice survived the infection (Figure 5e). Finally, to rule out MyD88 signaling within other pulmonary epithelial cells than CCs, we generated BM chimeric mice consisting of lethally irradiated CCSP-MyD $88^{\mathrm{ON}}$ mice reconstituted with $\mathrm{BM}$ from donor $\mathrm{WT}, \mathrm{CD} 11 \mathrm{c}-\mathrm{MyD} 88^{\mathrm{ON}}$, LysM-MyD88 ${ }^{\mathrm{ON}}$, or $\mathrm{MyD}^{\mathrm{OFF}}$ mice. WT $\rightarrow$ CCSP$\mathrm{MyD}_{8}{ }^{\mathrm{ON}}, \quad \mathrm{CD} 11 \mathrm{c}-\mathrm{MyD} 88^{\mathrm{ON}} \rightarrow \mathrm{CCSP}-\mathrm{MyD} 88^{\mathrm{ON}}, \quad$ and LysM-MyD88 ${ }^{\mathrm{ON}} \rightarrow \mathrm{CCSP}-\mathrm{MyD} 88^{\mathrm{ON}}$ mice showed $\geqslant 80 \%$ survival, whereas all $\mathrm{MyD} 88^{\mathrm{OFF}} \rightarrow \mathrm{CCSP}-\mathrm{MyD} 88^{\mathrm{ON}}$ mice succumbed to infection (Figure 5f), demonstrating that MyD88 signaling by CCs and CD11c ${ }^{+}$or $\mathrm{LysM}^{+}$cells is critically needed within the early phase of infection to rescue mice from an otherwise lethal outcome.
In summary, our data suggest that MyD88 mediated proinflammatory responses in lung epithelium, AMs, and PMNs are required and act in concert to control and clear S. pneumoniae infection.

\section{DISCUSSION}

The central importance of MyD88 signaling during $S$. pneumoniae infection for the induction of a protective immune response is demonstrated by the high susceptibility and early death of MyD88-deficient mice. ${ }^{31}$ In humans, polymorphisms in genes encoding TLRs have been associated with enhanced susceptibility to recurrent infection with pyogenic bacteria, in particular with $S$. pneumoniae. ${ }^{47}$ Mice with individual TLR or IL-1R knockouts were shown to have defects in early proinflammatory responses such as reduced cytokine and chemokine levels, delayed PMN recruitment, or less phagocytic activity within the first hours after S. pneumoniae infection. ${ }^{8,10,28,29}$ Nevertheless, these impaired initial responses had only partial consequences in establishing functional protection, since the majority of mutant mice survived the infection. Hence, single TLR or IL-1R deficiency has a limited importance in protection against $S$. pneumoniae. In contrast, MyD88, the central adapter protein for most TLRs and the IL-1R, is of utmost relevance during pneumococcal immunity, suggesting a certain degree of redundancy of pathogen recognition receptors. ${ }^{30,31}$

A variety of cell types of hematopoietic and non-hematopoietic origin express MyD88 to induce different inflammatory responses against several pathogens. ${ }^{35,36,48-51}$ Until now, the cell type-specific role of MyD88 signaling in control and eradication of pneumococci in the respiratory tract remains largely unexplored. In this study, we dissect MyD88 signaling from epithelial and hematopoietic cells during S. pneumoniae infection. To this end, we used BM chimeric mice, as well as conditional MyD88 knockin mouse lines, to gain further insights into the cell-specific contribution of MyD88 in initiating protective immune responses against pneumococcal infection. We demonstrate that both, the hematopoietic and non-hematopoietic compartment are critically dependent on MyD88 for sufficient control and eradication of lung invading S. pneumoniae.

PMN recruitment to the site of infection is a central prerequisite for bacterial eradication, since mice devoid of PMNs, by either using antibody depletion or MIP-2 neutralisation, display increased bacterial outgrowth and mortality upon S. pneumoniae or Klebsiella pneumoniae infection. ${ }^{12,52}$ Our study shows that functional MyD88 signaling in either the hematopoietic or the non-hematopoietic compartment has a critical role for the recruitment of PMNs by the release of PMN-attracting chemokines MIP-2 and KC. Airway epithelial cells have been shown to contribute to this process in a variety of experimental systems in vivo. ${ }^{22,34,50,53,54}$ In line with our data, pulmonary Pseudomonas aeruginosa infection or lipopolysaccharide (LPS) challenge using MyD88 BM chimeras, showed that lung epithelial cells expressed MIP-2 and/or KC to promote PMN recruitment in a 

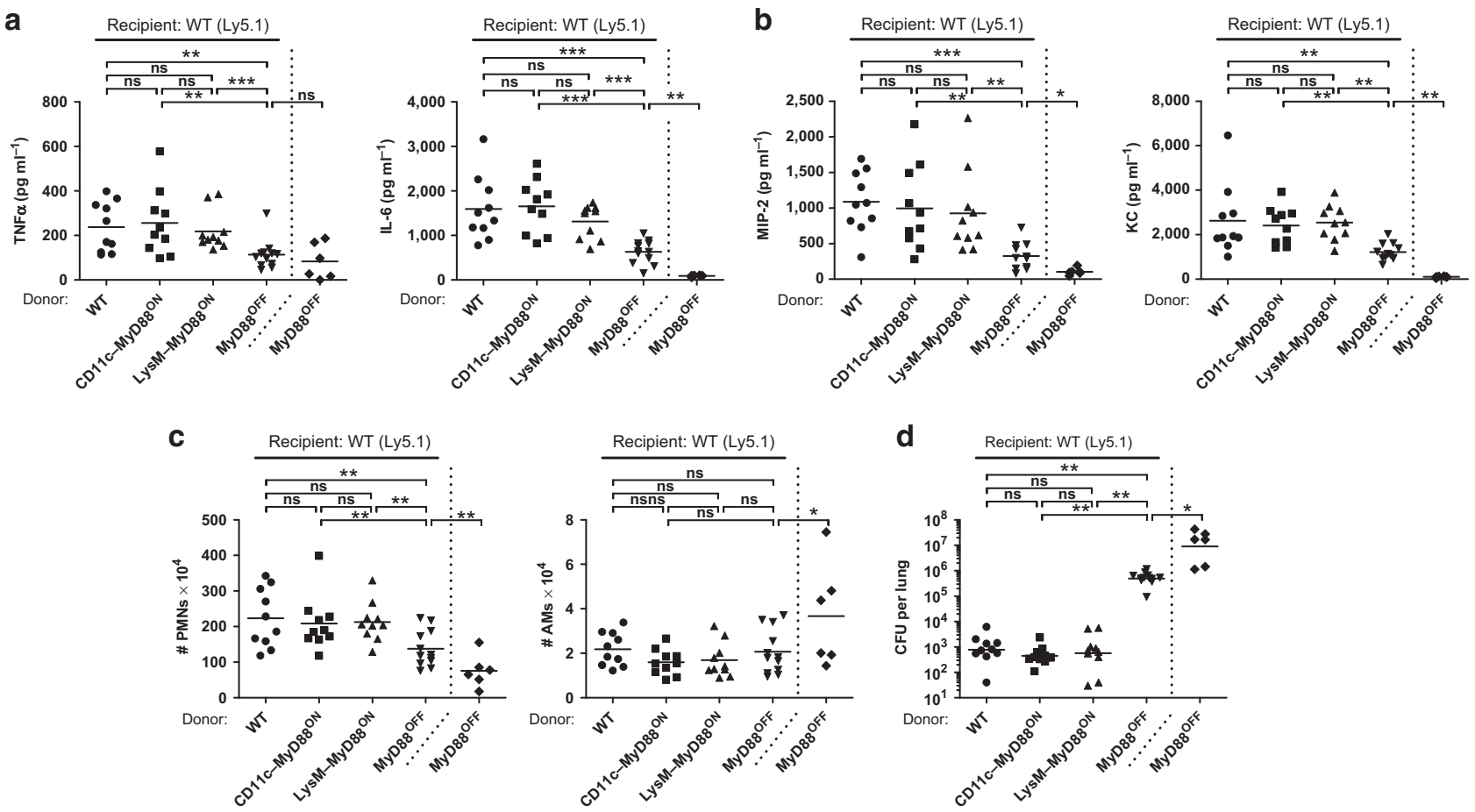

e
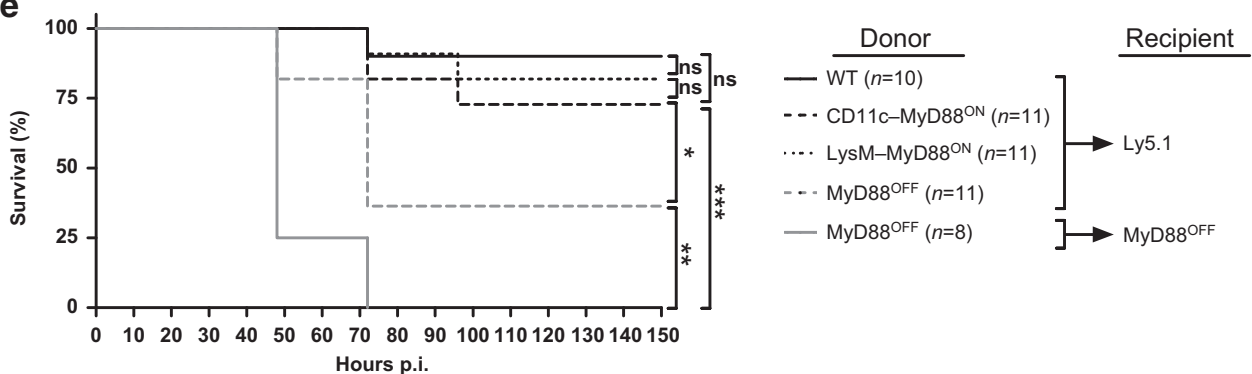

f

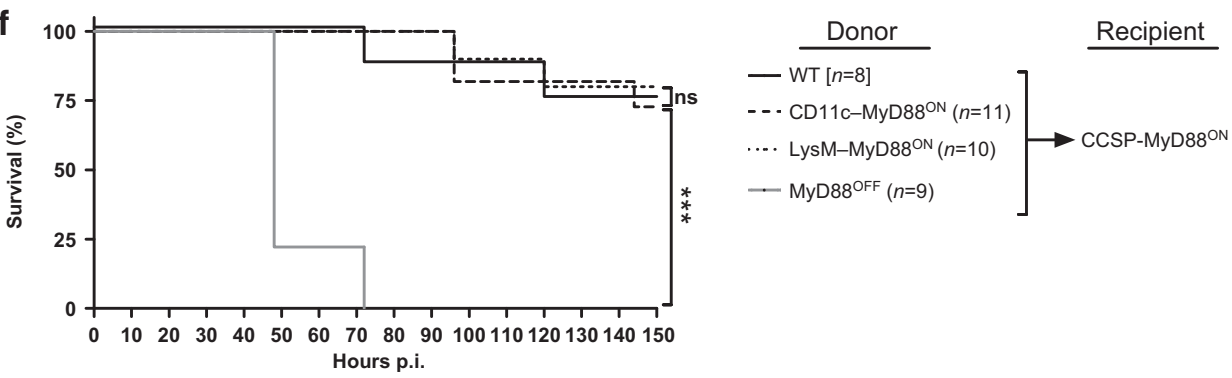

Figure 5 Lung epithelium, alveolar macrophages (AMs), and neutrophils (PMNs) act synergistically to clear pneumococcal infection in a myeloid differentiation factor 88 (MyD88)-dependent manner. Lethally irradiated recipient (a-e) wild type (WT) or (f) club cell secretory protein (CCSP)-MyD88 ${ }^{\mathrm{ON}}$ mice were reconstituted with bone marrow (BM) from donor CD11c-MyD88 ${ }^{\mathrm{N}}$, LysM-MyD88 ${ }^{\mathrm{ON}}$, or MyD88 ${ }^{\mathrm{OFF}}$ mice. After 8 weeks of reconstitution, mice were intranasally inoculated with either a high dose (a-d) or low dose (e,f) of Streptococcus pneumoniae. (a) Tumor necrosis factor $\alpha$ (TNF $\alpha$ ), interleukin-6 (IL-6), and (b) macrophage inflammatory protein 2 (MIP-2) and keratinocyte chemoattractant (KC) levels in total lung homogenates $18 \mathrm{~h}$ post infection (p.i.) measured by enzyme-linked immunosorbent assay (ELISA). (c) Fluorescence-activated cell sorting (FACS) analysis of CD11 $\mathrm{c}^{-} \mathrm{MHCII}^{-} \mathrm{CD} 1 \mathrm{~b}^{\text {high }}$

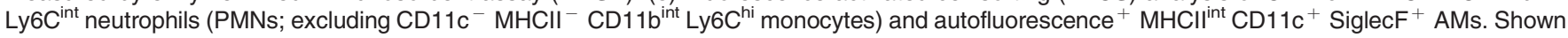
are total cell numbers in lungs from mice $18 \mathrm{~h}$ p.i. (d) $S$. pneumoniae burden in total lung homogenates $18 \mathrm{~h}$ p.i. (e,f) Survival curve of $S$. pneumoniaeinfected BM chimeric mice. (a-d) Data represent two pooled individual experiments with two to six mice per group. Data points depict individual mice. Bars indicate mean values. Statistics were calculated using Mann-Whitney test; ${ }^{*} P<0.05,{ }^{* \star} P<0.01$, and ${ }^{* * \star} P<0.001$. (e,f) Data are pooled from two individual experiments with three to six mice per group. Statistics were calculated using log-rank (Mantel-Cox) test; ${ }^{*} P<0.05,{ }^{* \star} P<0.01,{ }^{* * \star} P<0.001$, and ns, not significant.

MyD88-dependent manner. ${ }^{34,53}$ However, these experiments did not define which specific lung cell subsets are the most relevant for the chemokine secretion. Recently, it has been shown that the transformed murine CC cell line, C22, as well as ex vivo-isolated CCs can be stimulated to induce chemotactic cytokine production upon LPS treatment. ${ }^{21}$ Similarly, 
MyD88-dependent activation of CCs in airway inflammation with live $P$. aeruginosa was demonstrated. ${ }^{35}$ The authors used a MyD88 cDNA under the control of the rat CCSP promoter expressed in MyD88-deficient mice. P. aeruginosa-infected transgenic mice produced sufficient chemokines to recruit PMNs and to clear the infection. ${ }^{35}$ In contrast, our data show that upon S. pneumoniae infection, MyD88 signaling in CCs induces MIP-2 and KC production as well as PMN recruitment, albeit to a lower extent than in WT mice. Furthermore, CCSP-MyD88 ${ }^{\mathrm{ON}}$ mice failed to clear the infection. Differences between the two studies could be attributed to the different nature of the pathogens used. $P$. aeruginosa, a Gram-negative bacterium, activates a different set of pathogen recognition receptors than the Gram-positive S. pneumoniae. In addition, Mijares et al. used mice on a mixed genetic background not fully backcrossed onto the C57Bl/6J. In addition, in their study, Myd88 expression was under the control of the rat CCSP promoter, unlike our model, which uses the endogenous $M y d 88$ promoter elements and is therefore subjected to more physiological regulation. ${ }^{32}$ Besides CCs, other lung epithelial cells such as alveolar type I and II cells have been shown to contribute to PMN recruitment. ${ }^{54}$ We observed greater PMN recruitment in $\mathrm{BM}$ chimeric mice expressing MyD88 within the whole lung epithelium compared to CCSP-MyD88 ${ }^{\mathrm{ON}}$ mice, suggesting that chemokine secretion by several epithelial cells is needed for efficient chemotaxis. A certain level of redundancy might, however, exist between the non-hematopoietic and the hematopoietic compartment, as MyD88 signaling in CD11c ${ }^{+}$ or LysM ${ }^{+}$cells was sufficient to restore chemokine levels and thus PMN numbers to WT levels.

Proinflammatory cytokines, such as IL- 6 and TNF $\alpha$ promote not only the recruitment but also PMN activation. ${ }^{12,55,56}$ IL-6 plays an important role in the regulation of TNF $\alpha$, interferon- $\gamma$, and MPO secretion during pneumococcal infection, and deficiency in IL-6 leads to higher susceptibility and lethality. ${ }^{56}$ TNF $\alpha$ has been shown to be essential for the initiation of S. pneumoniae immunity, since depletion of $\mathrm{TNF} \alpha$ prior to infection increases the susceptibility and mortality rate. ${ }^{10,11,13}$ In addition, TNF $\alpha$ has been shown to compensate for host IL-1 type I receptor deficiency, demonstrating that $\mathrm{TNF} \alpha$ is more relevant for host defense against $S$. pneumoniae than IL-1. ${ }^{10}$ PMN-derived interferon- $\gamma$ was postulated to be critical in $S$. pneumoniae immunity by partially regulating the formation of neutrophil extracellular traps and inducing apoptosis, both contributing to bacterial clearance. ${ }^{57}$ However, we could not observe any MyD88-dependent differences in interferon- $\gamma$ production. Upon pneumococcal infection WT,

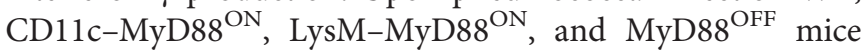
showed equally elevated interferon- $\gamma$ levels (data not shown). Here, we show that upon pneumococcal infection, secretion of TNF $\alpha$ unlike IL-6, is critically dependent on intrinsic MyD88 signaling in the myeloid cell compartment, as previously postulated for other bacterial infections. ${ }^{34,35}$

Recruited PMNs in CCSP-MyD88 ${ }^{\mathrm{ON}}$ mice showed no activation in terms of Tnfa, Il6, or Mip2 transcription. Moreover, low $\mathrm{MPO}^{+} \mathrm{PMN}$ numbers were observed in these mice. In contrast, PMNs from CD11c-MyD88 ${ }^{\mathrm{ON}}$ and LysM-MyD88 ${ }^{\mathrm{ON}}$ mice had increased transcript levels of proinflammatory cytokine genes along with high $\mathrm{MPO}^{+}$ PMN numbers, suggesting the requirement of MyD88 signaling in myeloid cells for full PMN activation. In particular, since MyD88 reactivation in AMs, but not in PMNs, was comparable between $\mathrm{CD} 11 \mathrm{c}-\mathrm{MyD} 88^{\mathrm{ON}}$ and $\mathrm{LysM}-\mathrm{MyD} 88^{\mathrm{ON}}$ mice, we cannot exclude indirect cytokine-mediated activation of PMNs independent of intrinsic MyD88 signaling, for example, via AM-derived IL-6. New and more specific Cre lines will therefore be needed to dissect the direct vs. indirect contribution of MyD88 signaling in the different myeloid cell populations.

Isolated primary $\mathrm{AMs}$ from $\mathrm{MyD} 88^{-/}$mice have been shown to have a reduced uptake of pneumococci, providing an explanation as to why pneumococcal outgrowth and systemic spread is strongly enhanced in mice deficient for MyD88 signaling in hematopoietic cells. ${ }^{28}$ In addition, upon S. pneumoniae infection, AMs have been shown to undergo apoptosis. This represents a critical host response that contributes to bacterial eradication and to the resolution of inflammation in the lungs. ${ }^{14,58}$ Caspase- 3 is a frequently activated protease in cell apoptosis and was shown to be relevant in $S$. pneumoniae-induced apoptosis in AMs in cooperation with TRAIL expression in PMNs. ${ }^{45}$ Our results show that MyD88 signaling in AMs critically contributes to the induction of caspase-3 expression and TRAIL might be involved in this process in a MyD88-dependent manner.

The key finding in our study, that MyD88 signaling solely in hematopoietic or non-hematopoietic cells was not sufficient to eradicate bacteria as in WT mice reveals the crucial interplay of both compartments and draws the focus on epithelial cell-derived mediators in pneumococcal clearance. Besides the release of chemokines and cytokines, lung epithelial cells secrete a large array of bacteriostatic or bactericidal molecules. These antimicrobial peptides protect from invading pathogens by several mechanisms, including opsonization, pore formation, or direct bacterial killing. ${ }^{59}$ Lung epithelial CCs secret several antimicrobial peptides, of which SP-D is consistently reported to be crucially involved in S. pneumoniae immunity. SP-D efficiently binds and aggregates pneumococci in vitro. ${ }^{40}$ Furthermore, SP-D-deficient mice as well as humans have an increased susceptibility to $S$. pneumoniae infection. ${ }^{26,27,41}$ Here, we demonstrate that MyD88 signaling in CCs, but not in myeloid cells, is required for the enhanced production of SP-D in response to $S$. pneumoniae infection. This may contribute to the clearance of pneumococci, as illustrated by BM chimeric mice with functional MyD88 signaling in lung epithelial cells and either $\mathrm{CD}_{11 \mathrm{c}^{+}}$or LysM ${ }^{+}$cells, where the bacterial burden was comparable to WT mice. Further studies will be necessary to analyze the contribution of other antimicrobial peptides such as serum amyloid A or surfactant protein A, which both have been shown to participate in antimicrobial defense of Gram-positive and -negative bacteria. ${ }^{40,50,60}$

Overall, our results suggest that AMs and PMNs provide essential chemokines and proinflammatory cytokines to recruit phagocytic immune cells, which are in turn partially dependent 
on epithelial cell-derived SP-D to entirely eradicate pulmonary pneumococci. We propose that AMs, PMNs, and the lung epithelium are critically dependent on MyD88 signaling and act in concert to coordinate innate immunity against S. pneumoniae. This might have direct implications for other lung infection models such as Staphylococcus aureus, $P$. aeruginosa, and Haemophilus influenzae, in which the bacterial burden is also critically controlled by myeloid and epithelial cells.

\section{METHODS}

Mice. All animal experiments were performed in compliance with the German animal protection law (TierSchG BGBI. I S. 1105; 25.05.1998), and approved by the Lower Saxony Committee on the Ethics of Animal Experiments as well as the responsible state office (Lower Saxony State Office of Consumer Protection and Food Safety). Mice were housed and handled in accordance with good animal practice as defined by the Federation of European Laboratory Animal Science Associations (FELASA) and the national animal welfare body Society of Laboratory Animals (GV-SOLAS). Mice were bred at the Twincore and Helmholtz Centre for Infection Research (HZI) animal facilities (Hannover and Braunschweig, Lower Saxony, Germany) (Hannover/Braunschweig) under specific pathogen-free conditions.

MyD $88^{-1-}$ mice were kindly provided by Shizuo Akira; ${ }^{61}$ MyD88 ${ }^{\text {OFF }}$ mice were provided by Bernhard Holzmann; ${ }^{36}$ RFP mice were provided by Hans Jörg Fehling; ${ }^{39} \mathrm{CD} 11 \mathrm{c}-\mathrm{Cre}$ mice were provided by Boris Reizis; ${ }^{42}$ LysM-Cre mice were provided by Irmgard Förster; ${ }^{43}$ and CCSP-Cre mice were provided by Francesco J DeMayo. ${ }^{37}$ All the mice used in the experiments were on the C57Bl/6J background, sex- and agematched and of 6-16 weeks of age. Mice denoted as WT mice in Figure 1 were $\mathrm{C} 57 \mathrm{Bl} / 6$ mice and in Figures 2-5 were from the CD11c-Cre or CCSP-Cre breeding.

To generate BM chimeras, mice were lethally irradiated with $9 \mathrm{~Gy}$. Donor BM cells were transferred intravenous (i.v.) the next day. Engraftment was assessed prior to the experiments 7-8 weeks later in the blood and during the experiment from total lung cells. After 8 weeks of reconstitution mice were taken for infection experiments.

Antibodies. The following antibodies were purchased from eBioscience (Heidelberg, Germany): APC-conjugated anti-mouse CD3E (clone: 145-2C11), APC-conjugated anti-mouse CD11b (clone: M1/70), APC eFluor 780-conjugated anti-mouse CD11b (clone: M1/ 70), eFluor 450-conjugated anti-mouse CD11b (clone: M1/70), APC eFluor 780-conjugated anti-mouse CD11c (clone: N418), Alexa Fluor 647-conjugated anti-mouse CD19 (clone: 1D3), PE-conjugated antimouse CD45.1 (clone: A20), APC-conjugated anti-mouse CD45.2 (clone: 104), PE-conjugated anti-mouse CD103 (clone: 2E7), PE Cyan 7-conjugated anti-mouse CD45R/B220 (clone: RA3-6B2), Alexa Fluor 647-conjugated anti-mouse Gr-1 (clone: RB6-8C5), FITC-conjugated anti-mouse MHCII (clone: M5/114.15.2), APC-conjugated antimouse MHCII (clone: M5/114.15.2), and APC-conjugated anti-mouse NK1.1 (clone: PK136); the following antibodies were purchased from BioLegend (Fell, Germany): Pacific Blue-conjugated anti-mouse CD103 (clone: 2E7), PE Cyan 7-conjugated anti-mouse Ly-6C (clone: HK1.4), and PE Cyan 7-conjugated anti-mouse Ly-6G (clone: 1A8); the following antibody was purchased from $\mathrm{BD}$ Pharmingen (Heidelberg, Germany): Brilliant Violet 421-conjugated anti-mouse Siglec-F (clone: E50-2440); the following antibodies were purchased from R\&D Systems (Wiesbaden, Germany): sheep anti-mouse SP-D and donkey horseradish peroxidase-conjugated anti-sheep IgG; the following antibody was purchased from Statens Serum Institut (Hillerod, Denmark): Neufeld pneumococcal antiserum type 4; the following antibody was purchased from Dako (Hamburg, Germany): polyclonal anti-MPO; the following antibodies were purchased from Dianova (Hamburg, Germany): biotinylated donkey anti-rabbit and donkey serum; the following antibody was purchased from antibodies online (Aachen, Germany): polyclonal anti-RFP; the following antibody was purchased from Life Technologies (Darmstadt, Germany): Alexa Fluor 555-conjugated donkey anti-rabbit.

Bacterial culture and infection. For infection studies, the S. pneumoniae TIGR4 strain and the luciferase-expressing $S$. pneumoniae T4X strain $^{31}$ (kindly provided by B Henriques-Normark) were used. S. pneumoniae was grown overnight from frozen bead stocks on blood agar plates at $37^{\circ} \mathrm{C}$ and $5 \% \mathrm{CO}_{2}$. The next day single colonies were inoculated into Todd Hewitt broth supplemented with $0.5 \%$ yeast extract and grown to mid-logarithmic phase $\left(\mathrm{OD}_{600}=0.3\right)$ resulting in $\sim 1 \times 10^{8} \mathrm{CFUs} \mathrm{ml}^{-1}$. Appropriate dilutions were made to obtain the desired infectious dose of $0.2 \times 10^{6} \mathrm{CFUs}$ for low-dose infections and $2 \times 10^{6}$ CFUs for high-dose infections. CFUs were confirmed by $\log _{10}$ serial dilutions of the inoculum cultured overnight on blood agar plates. Mice were anesthetized with ketamine (Bela-Pharm, Vechta, Germany)/ xylazine (CP-Pharma, Burgdorf, Germany), and a total of $20 \mu \mathrm{l}$ containing the desired CFU of $S$. pneumoniae in phosphate-buffered saline (PBS) were intranasally inoculated.

Isolation of lung cells and determination of bacterial burden. Mice were killed by $\mathrm{CO}_{2}$ asphyxiation, and perfused with $5 \mathrm{ml}$ ice-cold PBS. Lung lobes were separated from the trachea, chopped, and incubated in Roswell Park Memorial Institute (RPMI) 1640 GlutaMAX medium (Life/Gibco, Darmstadt, Germany) supplemented with 5\% fetal calf serum (Biochrom, Berlin, Germany), containing $2.2 \mathrm{mg} \mathrm{ml}^{-1}$ collagenase D (Sigma-Aldrich, Seelze, Germany) and $0.055 \mathrm{mg} \mathrm{ml}^{-1}$ DNase I (Roche, Mannheim, Germany) for $45 \mathrm{~min}$ at $37^{\circ} \mathrm{C}$. Digestion was stopped by addition of $10 \mathrm{~mm}$ ethylenediaminetetraacetic acid (EDTA, pH 7.5), and the lung cell suspension was passed through a 70$\mu \mathrm{m}$ cell strainer. Aliquots were taken for CFU determination, fluorescence-activated cell sorting (FACS) analysis, and enzymelinked immunosorbent assay (ELISA) of cytokines.

CFU determination was performed by $\log _{10}$ serial dilution of the aliquot and incubation on blood agar plates overnight at $37^{\circ} \mathrm{C}$ and $5 \%$ $\mathrm{CO}_{2}$. For FACS analysis, the aliquot was pelleted and red blood lysis $\left(150 \mathrm{~mm} \mathrm{NH}_{4} \mathrm{Cl}, 10 \mathrm{~mm} \mathrm{KHCO}_{3}\right.$, and 0.1 mm EDTA at $\mathrm{pH}$ 7.2-7.4) was performed for $2 \mathrm{~min}$ at room temperature and stopped by adding phosphate-bovine-azide (PBA) (2 mM EDTA, 0.5\% bovine serum albumin, and $0.02 \%$ sodium azide in PBS). Lung cells were washed with PBA, and then used for FACS staining. For ELISA, one volume of lung lysis buffer $\left(600 \mathrm{~mm} \mathrm{NaCl}, 4 \mathrm{~mm} \mathrm{MgCl}_{2} 6 \mathrm{H}_{2} \mathrm{O}, 4 \mathrm{~mm} \mathrm{CaCl}{ }_{2} 2 \mathrm{H}_{2} \mathrm{O}\right.$, 60 mm TRIS, 2\% Triton X-100 (Sigma-Aldrich, München, Germany), and complete protease inhibitor cocktail (Roche) at $\mathrm{pH}$ 7.4) was added to the aliquot, and incubated for $20 \mathrm{~min}$ at $4{ }^{\circ} \mathrm{C}$. Cell debris was pelleted, and the supernatant was used for ELISA. The concentrations of cytokines in lung homogenates were analyzed using the DuoSet ELISA kits (R\&D Systems) according to the manufacturer's instructions.

Flow cytometry. For cell surface marker staining, cells were preincubated with $1 \%$ Fc-block in PBA for 10 min on ice, and then washed and incubated with the indicated monoclonal antibody conjugates for $20 \mathrm{~min}$ on ice in a total volume of $50 \mu \mathrm{l}$ of PBA. Dead cells were excluded by 4',6-diamidin-2-phenylindol (DAPI; Sigma-Aldrich) or ethidium bromide monoazide (EMA; Sigma-Aldrich) staining, and cellular aggregates were excluded by side scatter pulse width. After the staining procedure cells were fixed in $200 \mu \mathrm{l}$ PBS containing $2 \%$ paraformaldehyde for $15 \mathrm{~min}$ on ice. After fixation cells were washed and resuspended in PBA and stored at $4{ }^{\circ} \mathrm{C}$ until FACS analysis. Samples were acquired on a LSRII (BD Biosciences, Heidelberg, Germany), and analyzed with FlowJo software (Tree Star, Ashland, OR).

Immunohistochemistry. Formalin-fixed lungs were embedded in paraffin, and $1-2 \mu \mathrm{m}$ sections were cut. Paraffin sections were deparaffinized and subjected to a heat-induced epitope retrieval step. 
Slides were rinsed in cool running water and washed in Tris-buffered saline ( $\mathrm{pH}$ 7.4) prior to incubation with polyclonal anti-MPO (Dako) or with Neufeld antiserum (Statens Serum Institut) for $30 \mathrm{~min}$ at room temperature and overnight at $4{ }^{\circ} \mathrm{C}$, respectively. After rinsing, sections stained for MPO were incubated for $30 \mathrm{~min}$ at room temperature with biotinylated donkey anti-rabbit (Dianova). For detection, Dako REAL Detection System, alkaline phosphatase/Red (Dako) was used. Alkaline phosphatase was revealed by Fast Red (Dako) as chromogen for $30 \mathrm{~min}$ at room temperature. For detection of pneumococci, Dako EnVision + System-HRP labelled polymer (Dako) was used. Horseradish peroxidase was revealed by diaminobenzidine as chromogen for $5 \mathrm{~min}$ at room temperature. Nuclei were counterstained with hematoxylin and slides were mounted with gelatine (Merck Millipore, Darmstadt, Germany).

For detection of RFP, $5 \mu \mathrm{m}$ sections of cryopreserved lungs were fixed in acetone and incubated with serum-free protein block (Dako). After tapping off the blocking solution, sections were incubated with polyclonal anti-RFP (antibodies online, Aachen) overnight at $4{ }^{\circ} \mathrm{C}$. Slides were rinsed, blocked with donkey serum (Dianova), and incubated with Alexa Fluor 555-conjugated donkey anti-rabbit (Life Technologies). Nuclei were counterstained with DAPI (SigmaAldrich), and slides were mounted with Fluoromount-G (eBioscience).

Negative controls were performed omitting primary antibodies. Images were acquired using an Axio Imager Z1 microscope (Carl Zeiss MicroImaging, Jena, Germany). Positive cells were quantified in 10 high power fields (h.p.f.; 1 h.p.f. $=0.237 \mathrm{~mm}^{2}$ ).

For evaluation of histomorphology, hematoxylin and eosin-stained paraffin sections were scored as follows. The perivascular, peribronchial, and interstitial infiltration of leukocytes was individually scored with 1 as minimal, 2 as mild, and 3 as severe infiltration. The overall inflammation score is the sum of the individual scores. All immunohistochemical evaluations were performed in a blinded manner.

In vivo imaging. Mice were anesthetized with isoflurane carried in $2 \%$ $\mathrm{O}_{2}$ and placed in a supine position in a chamber for imaging with the IVIS Spectrum CT (PerkinElmer, Waltham, MA). For detection of three-dimensional bioluminescent signal, anesthetized mice were first scanned by computed tomography and then $S$. pneumoniae-derived bioluminescence was detected by the charge-coupled device camera. Analysis was performed with Living Image 4.4 software (PerkinElmer).

BAL and western blot. Mice were killed by $\mathrm{CO}_{2}$ asphyxiation and $\mathrm{BAL}$ was collected by gently lavaging the lung two times with $0.7 \mathrm{ml}$ ice-cold PBS. BAL was centrifuged and the cell-free supernatant was stored at $-80{ }^{\circ} \mathrm{C}$ until western blot analysis. Total protein concentration in BAL was measured by bicinchoninic acid assay (Life Technologies). Equal amounts of total protein were separated by sodium dodecyl sulfate-polyacrylamide gel electrophoresis and transferred to polyvinylidene fluoride membrane (Merck Millipore). Immunoblotting was performed using sheep anti-mouse SP-D and donkey horseradish peroxidase-conjugated anti-sheep IgG, and detected using ECL prime (GE Healthcare, Freiburg, Germany). Chemoluminescence was detected with the ChemoStar Imager (Intas, Göttingen, Germany), and analyzed with ImageJ software (National Institutes of Health, Bethesda, MD).

RNA isolation and quantitative real-time PCR. Total RNA was isolated from FACS-sorted lung cells using the RNeasy Micro kit (Qiagen, Hilden, Germany) according to the manufacturer's instructions. cDNA was generated using SuperScript III reverse transcriptase with oligo $(\mathrm{dT})_{12-18}$ primer in the presence of RNaseOUT recombinant ribonuclease inhibitor (all Invitrogen, Darmstadt, Germany). Real-time PCR was carried out with iQ SYBR green supermix (Bio-Rad, Munich, Germany). Following primers were used: $\beta$-actin forward strand 5'-TGTTACCAACTGGGACGACA-3', reverse strand $5^{\prime}$-GGGGTGTTGAAGGTCTCAAA-3'; Myd88 forward strand $5^{\prime}$-AGAGCTGCTGGCCTTGTTAG-3', reverse strand
5'-TTCTCGGACTCCTGGTTCTG-3'; Trail forward strand 5'-GA AGACCTCAGAAAGTGGC-3', reverse strand 5'-GACCAGCTC TCCATTCCTA-3'; $\quad C c r 7$ forward strand $5^{\prime}$-GTGTGCTTCTG CCAAGATGA- $3^{\prime}$, reverse strand $5^{\prime}$-CCACGAAGCAGATGACA GAA-3'; Casp3 forward strand 5'-TCAGAGGCGACTACTGC CGGA- $3^{\prime}$, reverse strand $5^{\prime}$-CCACCGGTATCTTCTGGCAAGCC-3'; Tnfa forward strand $5^{\prime}$-CATCTTCTCAAAATTCGAGTGACAA-3', reverse strand $5^{\prime}$-TGGGAGTAGACAAGGTACAACCC-3'; Mip2 forward strand 5'-AGACAGAAGTCATAGCCACTCTCAAG-3', reverse strand $5^{\prime}$-CCTCCTTTCCAGGTCAGTTAGC-3'; Il6 forward strand $5^{\prime}$-TGTCTATACCACTTCACAAGTCGGAG-3', reverse strand $5^{\prime}$-GCACAACTCTTTTCTCATTTCCAC-3' (all primers were obtained from Eurofins MWG Operon, Hamburg, Germany). Samples were analyzed on a LightCycler 480 II (Roche). Relative gene expression was calculated as $2^{-\mathrm{Ct}(\text { target })} / 2^{-\mathrm{Ct}(\beta \text {-actin })}$.

Statistics. For survival curves, a log-rank (Mantel-Cox) test was performed. All other statistical analyses were performed using MannWhitney test unless additionally indicated. Analysis was performed using GraphPad Prism software (GraphPad software, La Jolla, CA); ${ }^{\star} P<0.05,{ }^{\star *} P<0.01$, and ${ }^{\star * *} P<0.001$.

Ethics statement. All animal experiments were approved by the Lower Saxony Committee on the Ethics of Animal Experiments as well as the responsible state office (Lower Saxony State Office of Consumer Protection and Food Safety) under the permit numbers 33.9-4250204-09/1785 and 33.12-42502-04-12/0910.

SUPPLEMENTARY MATERIAL is linked to the online version of the paper at http://www.nature.com/mi

\section{ACKNOWLEDGMENTS}

We thank M Swallow and P Ghorbani for critical reading of the manuscript, and $\mathrm{F}$ Kruse, M Swallow, M Gohmert, and M Thiele for their excellent technical help. We also acknowledge the assistance of the Cell Sorting Core Facility of the Hannover Medical School supported in part by Braukmann-Wittenberg-Herz-Stiftung and Deutsche Forschungsgemeinschaft. MD was supported by the International Research Training Group 1273 funded by the German Research Foundation (DFG).

\section{DISCLOSURE}

The authors declared no conflict of interest.

c 2016 Official journal of the Society for Mucosal Immunology

\section{REFERENCES}

1. Bogaert, D., De Groot, R. \& Hermans, P.W. Streptococcus pneumoniae colonisation: the key to pneumococcal disease. Lancet Infect. Dis. 4, 144154 (2004)

2. Kadioglu, A. \& Andrew, P.W. The innate immune response to pneumococcal lung infection: the untold story. Trends Immunol. 25, 143-149 (2004).

3. Aliprantis, A.O. et al. Cell activation and apoptosis by bacterial lipoproteins through toll-like receptor-2. Science 285, 736-739 (1999).

4. Schwandner, R., Dziarski, R., Wesche, H., Rothe, M. \& Kirschning, C.J. Peptidoglycan- and lipoteichoic acid-induced cell activation is mediated by toll-like receptor 2. J. Biol. Chem. 274, 17406-17409 (1999).

5. Heeg, K., Sparwasser, T., Lipford, G.B., Hacker, H., Zimmermann, S. \& Wagner, $\mathrm{H}$. Bacterial DNA as an evolutionary conserved ligand signalling danger of infection to immune cells. Eur. J. Clin. Microbiol. Infect. Dis. 17, 464-469 (1998).

6. Hemmi, H. et al. A Toll-like receptor recognizes bacterial DNA. Nature 408, 740-745 (2000).

7. Molloy, S. Pneumolysin: stimulating protection. Nat. Rev. Microbiol. 9, 4 (2011).

8. Malley, R. et al. Recognition of pneumolysin by Toll-like receptor 4 confers resistance to pneumococcal infection. Proc. Natl. Acad. Sci. USA 100, 1966-1971 (2003). 
9. Aberdein, J.D., Cole, J., Bewley, M.A., Marriott, H.M. \& Dockrell, D.H. Alveolar macrophages in pulmonary host defence the unrecognized role of apoptosis as a mechanism of intracellular bacterial killing. Clin. Exp. Immunol. 174, 193-202 (2013).

10. Rijneveld, A.W., Florquin, S., Branger, J., Speelman, P., Van Deventer, S.J. \& van der Poll, T. TNF-alpha compensates for the impaired host defense of IL-1 type I receptor-deficient mice during pneumococcal pneumonia. J. Immunol. 167, 5240-5246 (2001).

11. O'Brien, D.P., Briles, D.E., Szalai, A.J., Tu, A.H., Sanz, I. \& Nahm, M.H. Tumor necrosis factor alpha receptor $\mathrm{I}$ is important for survival from Streptococcus pneumoniae infections. Infect. Immun. 67, 595-601 (1999).

12. Greenberger, M.J et al. Neutralization of macrophage inflammatory protein-2 attenuates neutrophil recruitment and bacterial clearance in murine Klebsiella pneumonia. J. Infect. Dis. 173, 159-165 (1996).

13. van der Poll, T., Keogh, C.V., Buurman, W.A. \& Lowry, S.F. Passive immunization against tumor necrosis factor-alpha impairs host defense during pneumococcal pneumonia in mice. Am. J. Respir. Crit. Care Med. 155, 603-608 (1997).

14. Knapp, S. et al. Alveolar macrophages have a protective antiinflammatory role during murine pneumococcal pneumonia. Am. J. Respir. Crit. Care Med. 167, 171-179 (2003).

15. Standish, A.J. \& Weiser, J.N. Human neutrophils kill Streptococcus pneumoniae via serine proteases. J. Immunol. 183, 2602-2609 (2009).

16. Guilliams, M., Lambrecht, B.N. \& Hammad, H. Division of labor between lung dendritic cells and macrophages in the defense against pulmonary infections. Mucosal Immunol. 6, 464-473 (2013).

17. Sung, S.S., Fu, S.M., Rose, C.E. Jr, Gaskin, F., Ju, S.T. \& Beaty, S.R. A major lung CD103 (alphaE)-beta7 integrin-positive epithelial dendritic cell population expressing Langerin and tight junction proteins. J. Immunol. 176, 2161-2172 (2006).

18. Colino, J., Shen, Y. \& Snapper, C.M. Dendritic cells pulsed with intact Streptococcus pneumoniae elicit both protein- and polysaccharidespecific immunoglobulin isotype responses in vivo through distinct mechanisms. J. Exp. Med. 195, 1-13 (2002).

19. Hippenstiel, S., Opitz, B., Schmeck, B. \& Suttorp, N. Lung epithelium as a sentinel and effector system in pneumonia-molecular mechanisms of pathogen recognition and signal transduction. Respir. Res. 7, 97 (2006).

20. Tomita, T., Sakurai, Y., Ishibashi, S. \& Maru, Y. Imbalance of Clara cellmediated homeostatic inflammation is involved in lung metastasis. Oncogene 30, 3429-3439 (2011).

21. Elizur, A., Adair-Kirk, T.L., Kelley, D.G., Griffin, G.L., deMello, D.E. \& Senior, R.M. Clara cells impact the pulmonary innate immune response to LPS. Am. J. Physiol. Lung Cell. Mol. Physiol. 293, L383L392 (2007).

22. Poynter, M.E., Irvin, C.G. \& Janssen-Heininger, Y.M. A prominent role for airway epithelial NF-kappa B activation in lipopolysaccharide-induced airway inflammation. J. Immunol. 170, 6257-6265 (2003).

23. Crouch, E., Parghi, D., Kuan, S.F. \& Persson, A. Surfactant protein D: subcellular localization in nonciliated bronchiolar epithelial cells. Am. J. Physiol. 263, L60-L66 (1992).

24. Wong, C.J., Akiyama, J., Allen, L. \& Hawgood, S. Localization and developmental expression of surfactant proteins $D$ and $A$ in the respiratory tract of the mouse. Pediatr. Res. 39, 930-937 (1996).

25. Botas, C. et al. Altered surfactant homeostasis and alveolar type II cell morphology in mice lacking surfactant protein D. Proc. Natl. Acad. Sci. USA 95, 11869-11874 (1998).

26. Griese, M., Steinecker, M., Schumacher, S., Braun, A., Lohse, P. \& Heinrich, $\mathrm{S}$. Children with absent surfactant protein $\mathrm{D}$ in bronchoalveolar lavage have more frequently pneumonia. Pediatr. Allergy Immunol. 19, 639-647 (2008).

27. Lingappa, J.R. et al. Identifying host genetic risk factors in the context of public health surveillance for invasive pneumococcal disease. PLoS One 6, e23413 (2011).

28. Albiger, B. et al. Toll-like receptor 9 acts at an early stage in host defence against pneumococcal infection. Cell. Microbiol. 9, 633-644 (2007).

29. Knapp, S. et al. Toll-like receptor 2 plays a role in the early inflammatory response to murine pneumococcal pneumonia but does not contribute to antibacterial defense. J. Immunol. 172, 3132-3138 (2004).

30. Akira, S. \& Takeda, K. Toll-like receptor signalling. Nat. Rev. Immunol. 4, 499-511 (2004).
31. Albiger, B. et al. Myeloid differentiation factor 88-dependent signalling controls bacterial growth during colonization and systemic pneumococcal disease in mice. Cell. Microbiol. 7, 1603-1615 (2005).

32. Arnold-Schrauf, C., Berod, L. \& Sparwasser, T. Dendritic cell specific targeting of MyD88 signalling pathways in vivo. Eur. J. Immunol. 45, 32-39 (2015).

33. Sandgren, A., Albiger, B., Orihuela, C.J., Tuomanen, E., Normark, S. \& Henriques-Normark, B. Virulence in mice of pneumococcal clonal types with known invasive disease potential in humans. J. Infect. Dis. 192, 791-800 (2005).

34. Hajjar, A.M., Harowicz, H., Liggitt, H.D., Fink, P.J., Wilson, C.B. \& Skerrett, S.J. An essential role for non-bone marrow-derived cells in control of Pseudomonas aeruginosa pneumonia. Am. J. Respir. Cell Mol. Biol. 33, 470-475 (2005).

35. Mijares, L.A., Wangdi, T., Sokol, C., Homer, R., Medzhitov, R. \& Kazmierczak, B.I. Airway epithelial MyD88 restores control of Pseudomonas aeruginosa murine infection via an IL-1-dependent pathway. J. Immunol. 186, 7080-7088 (2011).

36. Gais, P. et al. Cutting edge: divergent cell-specific functions of MyD88 for inflammatory responses and organ injury in septic peritonitis. J. Immunol. 188, 5833-5837 (2012).

37. Li, H., Cho, S.N., Evans, C.M., Dickey, B.F., Jeong, J.W. \& DeMayo, F.J. Cre-mediated recombination in mouse Clara cells. Genesis 46, 300-307 (2008).

38. Lau, D. et al. Myeloperoxidase mediates neutrophil activation by association with CD11b/CD18 integrins. Proc. Natl. Acad. Sci. USA 102, 431-436 (2005).

39. Luche, H., Weber, O., Nageswara Rao, T., Blum, C. \& Fehling, H.J. Faithful activation of an extra-bright red fluorescent protein in "knock-in" Crereporter mice ideally suited for lineage tracing studies. Eur. J. Immunol. 37 , 43-53 (2007).

40. Hartshorn, K.L. et al. Pulmonary surfactant proteins $A$ and D enhance neutrophil uptake of bacteria. Am. J. Physiol. 274, L958-L969 (1998).

41. Jounblat, R., Clark, H., Eggleton, P., Hawgood, S., Andrew, P.W. \& Kadioglu, A. The role of surfactant protein $D$ in the colonisation of the respiratory tract and onset of bacteraemia during pneumococcal pneumonia. Respir. Res. 6, 126 (2005).

42. Caton, M.L., Smith-Raska, M.R. \& Reizis, B. Notch-RBP-J signaling controls the homeostasis of CD8- dendritic cells in the spleen. J. Exp. Med. 204, 1653-1664 (2007).

43. Clausen, B.E., Burkhardt, C., Reith, W., Renkawitz, R. \& Forster, I. Conditional gene targeting in macrophages and granulocytes using LysMcre mice. Transgenic Res. 8, 265-277 (1999).

44. Vermaelen, K. \& Pauwels, R. Accurate and simple discrimination of mouse pulmonary dendritic cell and macrophage populations by flow cytometry: methodology and new insights. Cytometry A 61, 170-177 (2004).

45. Steinwede, K. et al. TNF-related apoptosis-inducing ligand (TRAIL) exerts therapeutic efficacy for the treatment of pneumococcal pneumonia in mice. J. Exp. Med. 209, 1937-1952 (2012).

46. Nakano, $\mathrm{H}$. et al. Migratory properties of pulmonary dendritic cells are determined by their developmental lineage. Mucosal Immunol. 6, 678-691 (2013).

47. Netea, M.G., Wijmenga, C. \& O'Neill, L.A. Genetic variation in Toll-like receptors and disease susceptibility. Nat. Immunol. 13, 535-542 (2012).

48. Arnold-Schrauf, C. et al. Dendritic cells coordinate innate immunity via MyD88 signaling to control Listeria monocytogenes infection. Cell Rep. 6, 698-708 (2014).

49. Berod, L. et al. MyD88 signalling in myeloid cells is sufficient to prevent chronic mycobacterial infection. Eur. J. Immunol. 44, 13991409 (2014).

50. Cleaver, J.O. et al. Lung epithelial cells are essential effectors of inducible resistance to pneumonia. Mucosal Immunol. 7, 78-88 (2014).

51. Hammad, H., Chieppa, M., Perros, F., Willart, M.A., Germain, R.N. \& Lambrecht, B.N. House dust mite allergen induces asthma via Toll-like receptor 4 triggering of airway structural cells. Nat. Med. 15, 410-416 (2009).

52. Matthias, K.A., Roche, A.M., Standish, A.J., Shchepetov, M. \& Weiser, J.N. Neutrophil-toxin interactions promote antigen delivery and mucosal 
clearance of Streptococcus pneumoniae. J. Immunol. 180, 6246-6254 (2008).

53. Noulin, N. et al. Both hemopoietic and resident cells are required for MyD88-dependent pulmonary inflammatory response to inhaled endotoxin. J. Immunol. 175, 6861-6869 (2005).

54. Yamamoto, K. et al. Roles of lung epithelium in neutrophil recruitment during pneumococcal pneumonia. Am. J. Respir. Cell Mol. Biol. 50, 253262 (2014).

55. Lukacs, N.W., Strieter, R.M., Chensue, S.W., Widmer, M. \& Kunkel, S.L. TNF-alpha mediates recruitment of neutrophils and eosinophils during airway inflammation. J. Immunol. 154, 5411-5417 (1995).

56. van der Poll, T., Keogh, C.V., Guirao, X., Buurman, W.A., Kopf, M. \& Lowry, S.F. Interleukin-6 gene-deficient mice show impaired defense against pneumococcal pneumonia. J. Infect. Dis. 176, 439-444 (1997).

57. Gomez, J.C. et al. Mechanisms of interferon-gamma production by neutrophils and its function during Streptococcus pneumoniae pneumonia. Am. J. Respir. Cell Mol. Biol. 52, 349-364 (2015).

58. Dockrell, D.H. et al. Alveolar macrophage apoptosis contributes to pneumococcal clearance in a resolving model of pulmonary infection. J. Immunol. 171, 5380-5388 (2003).
59. Bals, R. \& Hiemstra, P.S. Innate immunity in the lung: how epithelial cells fight against respiratory pathogens. Eur. Respir. J. 23, 327-333 (2004).

60. Shah, C., Hari-Dass, R. \& Raynes, J.G. Serum amyloid A is an innate immune opsonin for Gram-negative bacteria. Blood 108, 1751-1757 (2006).

61. Adachi, O. et al. Targeted disruption of the MyD88 gene results in loss of IL-1- and IL-18-mediated function. Immunity 9, 143-150 (1998).

This work is licensed under a Creative Commons Attribution-NonCommercial-ShareAlike 4.0 International License. The images or other third party material in this article are included in the article's Creative Commons license, unlessindicated otherwise in thecreditline; if the material is not included under the Creative Commons license, users will need to obtain permission from the license holder to reproduce the material. To view a copy of this license, visit http:// creativecommons.org/licenses/by-nc-sa/4.0/ 